\title{
Urolithin A suppresses high glucose-induced neuronal amyloidogenesis by modulating TGM2-dependent ER-mitochondria contacts and calcium homeostasis
}

\author{
Hyun Jik Lee $e^{1,2} \cdot$ Young Hyun Jung ${ }^{3} \cdot$ Gee Euhn Choi ${ }^{3} \cdot$ Jun Sung Kim $\mathbb{D}^{3} \cdot$ Chang Woo Chae $^{3} \cdot$ Jae Ryong Lim ${ }^{3}$. \\ Seo Yihl Kim ${ }^{3} \cdot$ Jee Hyeon Yoon ${ }^{3} \cdot$ Ji Hyeon $\mathrm{Cho}^{3} \cdot$ Sei-Jung Lee $\mathbb{C}^{4} \cdot$ Ho Jae $\mathrm{Han}^{3}$
}

Received: 9 March 2020 / Revised: 6 July 2020 / Accepted: 10 July 2020 / Published online: 23 July 2020

(c) The Author(s), under exclusive licence to ADMC Associazione Differenziamento e Morte Cellulare 2020

\begin{abstract}
Hyperglycemia in diabetes mellitus (DM) patients is a causative factor for amyloidogenesis and induces neuropathological changes, such as impaired neuronal integrity, neurodegeneration, and cognitive impairment. Regulation of mitochondrial calcium influx from the endoplasmic reticulum (ER) is considered a promising strategy for the prevention of mitochondrial ROS (mtROS) accumulation that occurs in the Alzheimer's disease (AD)-associated pathogenesis in DM patients. Among the metabolites of ellagitannins that are produced in the gut microbiome, urolithin A has received an increasing amount of attention as a novel candidate with anti-oxidative and neuroprotective effects in AD. Here, we investigated the effect of urolithin A on high glucose-induced amyloidogenesis caused by mitochondrial calcium dysregulation and mtROS accumulation resulting in neuronal degeneration. We also identified the mechanism related to mitochondria-associated ER membrane (MAM) formation. We found that urolithin A-lowered mitochondrial calcium influx significantly alleviated high glucose-induced mtROS accumulation and expression of amyloid beta $(\mathrm{A} \beta)$-producing enzymes, such as amyloid precursor protein (APP) and $\beta$-secretase-1 (BACE1), as well as A $\beta$ production. Urolithin A injections in a streptozotocin (STZ)induced diabetic mouse model alleviated APP and BACE1 expressions, Tau phosphorylation, A $\beta$ deposition, and cognitive impairment. In addition, high glucose stimulated MAM formation and transglutaminase type 2 (TGM2) expression. We first discovered that urolithin A significantly reduced high glucose-induced TGM2 expression. In addition, disruption of the AIP-AhR complex was involved in urolithin A-mediated suppression of high glucose-induced TGM2 expression. Markedly, TGM2 silencing inhibited inositol 1, 4, 5-trisphosphate receptor type 1 (IP3R1)-voltage-dependent anion-selective channel protein 1 (VDAC1) interactions and prevented high glucose-induced mitochondrial calcium influx and mtROS accumulation. We also found that urolithin $\mathrm{A}$ or TGM2 silencing prevented $\mathrm{A} \beta$-induced mitochondrial calcium influx, mtROS accumulation, Tau phosphorylation, and cell death in neuronal cells. In conclusion, we suggest that urolithin A is a promising candidate for the development of therapies to prevent DM-associated AD pathogenesis by reducing TGM2dependent MAM formation and maintaining mitochondrial calcium and ROS homeostasis.
\end{abstract}

These authors contributed equally: Hyun Jik Lee, Young Hyun Jung

Edited by: M. Piacentini

Supplementary information The online version of this article (https:// doi.org/10.1038/s41418-020-0593-1) contains supplementary material, which is available to authorized users.

Ho Jae Han

hjhan@snu.ac.kr

Extended author information available on the last page of the article

\section{Introduction}

Hyperglycemia in diabetes mellitus (DM) patients is a key pathogenic factor that contributes to amyloidogenesisinduced neuropathological changes, such as impaired neuronal integrity, neurodegeneration, and cognitive impairment [1, 2]. In addition, previous studies showed that a high glucose environment increases amyloid precursor protein (APP) accumulation and amyloid beta $(\mathrm{A} \beta)$ plaque formation in neuronal cells $[3,4]$. Increased expression of the APP processing enzyme $\beta$-secretase 1 (BACE1) by high glucose is critical for neuronal $A \beta$ production, and the inactivation of BACE1 alleviates A $\beta$-driven Alzheimer's 
disease (AD)-like pathologies, such as synaptic and memory deficits [5-7]. It is well known that hyperglycemiainduced diabetic complications, including cognitive impairment and executive functions, are mainly related to oxidative stress $[8,9]$. Mitochondrial reactive oxygen species (mtROS) play key roles in the pathogenesis of AD by promoting mitochondrial dysfunction, damage to RNA and DNA, lipid peroxidation, and $A \beta$ oxidation [10]. Because mtROS are involved in amyloidogenesis and neuronal apoptosis, the suppression of mtROS accumulation under hyperglycemic conditions is a promising strategy for the prevention of the $\mathrm{AD}$-associated pathogenesis in $\mathrm{DM}$ patients $[11,12]$.

Dysregulation of mitochondrial calcium homeostasis by hyperglycemia is a risk factor for mtROS accumulation, which leads to diabetic neuropathy $[13,14]$. A mitochondria-associated endoplasmic reticulum (ER) membrane (MAM) is a site of physiological communication between mitochondria and the ER, which controls cellular metabolism and calcium transport from the ER to mitochondria $[15,16]$. A study revealed that an impaired mitochondrial calcium efflux capacity in a $3 \times \mathrm{Tg}$-AD mouse model contributed to superoxide accumulation, metabolic dysfunction, and neuronal degeneration, suggesting calcium dysregulation of mitochondria as a therapeutic target in $\mathrm{AD}$ treatment [17]. Indeed, significant increases in MAM function and mitochondria-ER communications in presenilin-mutant cells and fibroblasts from familial and sporadic AD patients have been reported [18]. A $\beta$-peptideincreased MAM-dependent calcium transport is mediated by voltage-dependent anion-selective channel protein 1 (VDAC1)-inositol 1, 4, 5-trisphosphate receptor (IP3R) bridge formation in primary hippocampal neurons, which is involved in AD [19]. Regulatory elements of the MAM that modulate IP3R-VDAC1 interactions have been reported, such as GRP75, Bax, Bcl2, and TGM2 [20-22]. Furthermore, the importance of MAM was highlighted in studies that showed VDAC1 and mitochondrial calcium uniporter (MCU) inhibition significantly reversed high glucoseinduced mtROS accumulation in HepG2 cells and $\beta$-cell dysfunction in type 2 diabetes (T2D) islets [23, 24]. These findings suggest a close relationship between MAM-mediated mitochondrial calcium influx and mtROS accumulation in high glucose conditions. Therefore, MAMregulated mitochondrial calcium influx may be a potential therapeutic target to treat hyperglycemia-related $\mathrm{AD}$ pathogenesis.

3,8-Dihydroxy-urolithin (urolithin A) is a gut microbiome-produced end-stage metabolite of ellagitannins that is associated with the positive effects of ellagitanninrich products on chronic diseases, such as DM and neurodegenerative diseases [25]. The bioavailability of urolithins derived from ellagitannin and ellagic acid is dependent on individual gut microbiome composition [26, 27]. Indeed, previous researchers reported that urolithin conversion from ellagic acid is dependent on several identified species (Clostridium coccoides and the Eggerthellacae family) in human gut microbiota [26-28]. Many studies investigating the bioactivity of urolithins showed that urolithin $\mathrm{A}$ is the most potent metabolite among its precursor ellagitannin and other urolithins $[29,30]$. In silico computational studies revealed that urolithins are brain absorbable metabolites that can penetrate the blood-brain barrier and contribute to the alleviation of $A \beta$ (1-42)-induced neurotoxicity [31]. In addition, experimental evidence for ellagitannin-derived urolithin A deposition in the brain of a Parkinson's disease rat model has been presented [32]. Other researchers also reported that urolithin A exhibits a protective effect on oxidative stress-induced inflammation and apoptosis in neuronal and microglial cells [33-35]. Furthermore, urolithin A prolonged the lifespan of Caenorhabditis elegans and improved the exercise capacity of rodent models by suppressing mitochondrial oxygen consumption metabolism [36]. Despite this anti-oxidative action of urolithin A, the effects of urolithin A on MAM formation and mitochondrial calcium influx from the ER regulating mtROS generation in neuronal cells under high glucose conditions have not been reported yet. Here, we investigated the ability of urolithin A to reduce high glucose-induced amyloidogenesis through modulation of mitochondrial calcium influx and mtROS in neuronal cells. We also aimed to identify the underlying regulatory mechanisms.

\section{Results}

\section{Effect of urolithin A on high glucose-induced mitochondrial calcium influx and mtROS accumulation}

First, we investigated the effect of ellagic acid and urolithins on the cell viability of SH-SY5Y cells under a high glucose condition. The pretreatment of urolithin A but not urolithin $\mathrm{B}$, urolithin $\mathrm{C}$, or urolithin $\mathrm{D}$ significantly decreased the high glucose-induced LDH release level (Fig. 1a). The LDH release level in the urolithin A pretreatment with high glucose group was the lowest compared with other pretreatment groups (Fig. 1a). In the WST-1 cell viability assay, we confirmed that the viability of urolithin A-pretreated SHSY5Y cells with high glucose was higher than that of vehicle-pretreated SH-SY5Y cells with high glucose (Supplementary Fig. 1). MitoSOX staining assay showed that urolithin A pretreatment decreased high glucose-induced mtROS levels (Fig. 1b). However, Fig. 1b showed nonsignificant effects of ellagic acid and other urolithins (urolithin $\mathrm{B}, \mathrm{C}$, and $\mathrm{D}$ ). Based upon our findings presenting the 


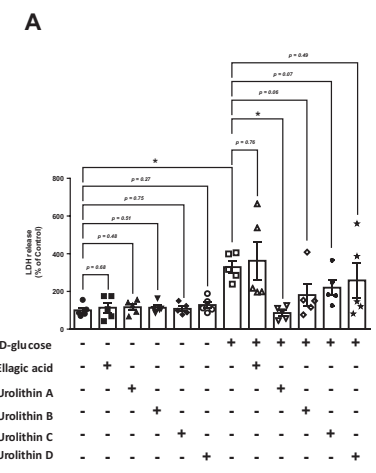

B

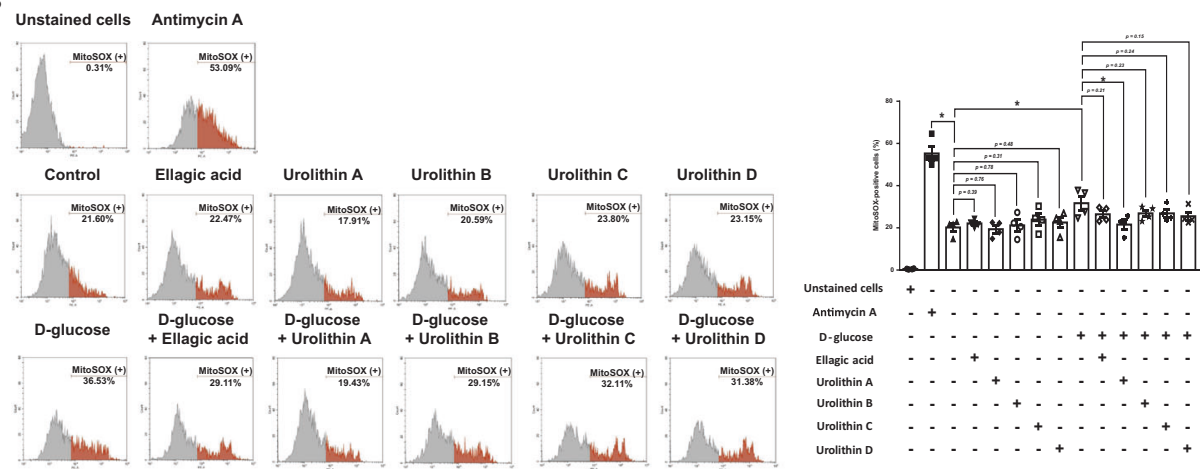

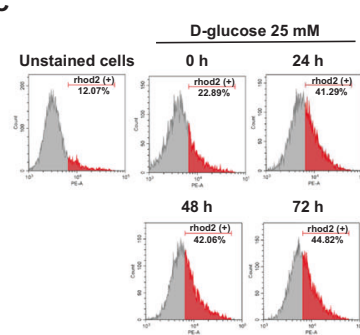

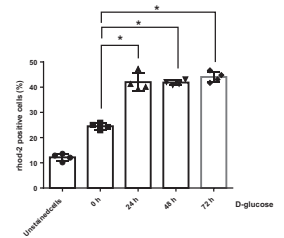

$\mathbf{F}$

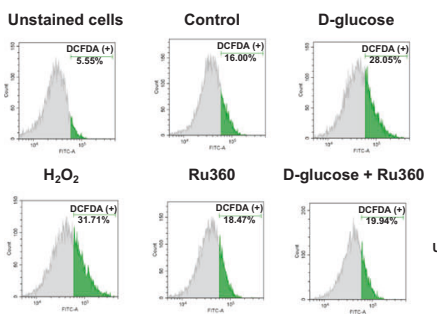

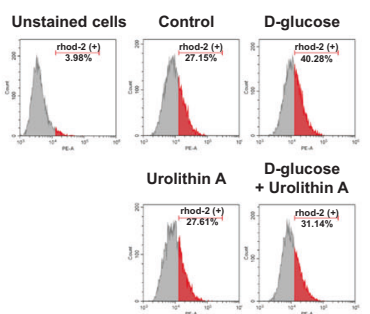
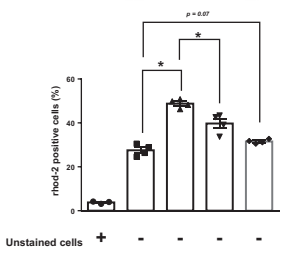

D-glucose $-\quad++\cdot$

Urolithin A - - ++

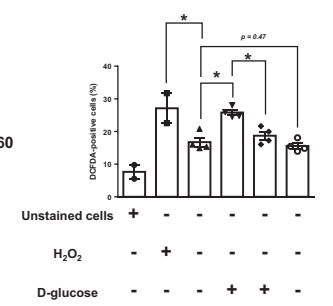

D-glucose $-\quad-\quad++$

Fig. 1 Effect of urolithins on mitochondrial calcium, ROS in neuronal cells under high glucose condition. a SH-SY5Y cells were treated with $100 \mathrm{nM}$ of ellagic acid, urolithin A, B, C, and D for 30 min prior to high glucose exposure ( $25 \mathrm{mM}$ of D-glucose) for $72 \mathrm{~h}$. LDH released from apoptotic cells in supernatant was analyzed with LDH assay kit, $n=5$. b Cells were pretreated with ellagic acid (100 $\mathrm{nM})$ or urolithin $\mathrm{A}(100 \mathrm{nM})$ or urolithin $\mathrm{B}(100 \mathrm{nM})$ or urolithin C $(100 \mathrm{nM})$ or urolithin $\mathrm{D}(100 \mathrm{nM})$ for 30 min prior to high glucose (25 $\mathrm{mM})$ treatment for $48 \mathrm{~h}$. Antimycin $\mathrm{A}(10 \mu \mathrm{M})$ was used for inducing mtROS accumulation to make a positive control for analysis. Cells stained with MitoSOX $(1 \mu \mathrm{M})$ for $20 \mathrm{~min}$ and MitoSOX-positive cells were analyzed with flow cytometer, $n=4$. c Cells treated with high glucose at the timepoint $(0-72 \mathrm{~h})$. Mitochondrial calcium levels were measured by staining the cells with rhod-2 fluorescent dye $(2 \mu \mathrm{M})$ for $20 \mathrm{~min}$, and rhod-2-positive cells were analyzed with flow cytometer, $n=4$. Unstained cells were used as a negative control. d, e Cells were

$\mathrm{E}$
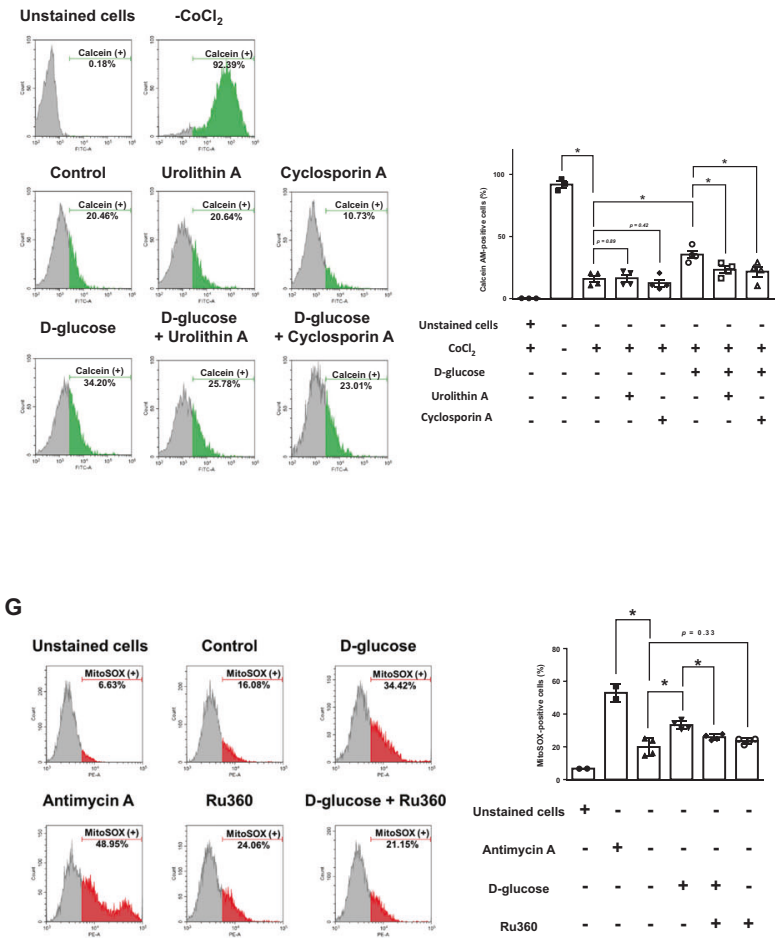

exposed with urolithin $\mathrm{A}(100 \mathrm{nM})$ for 30 min prior to high glucose for $48 \mathrm{~h}$. d rhod-2-positive cells are shown as percentages of the gated cells, $n=4$. e Mitochondrial permeability transition pore (mPTP) assay was performed and analyzed by flow cytometer. Cells were pretreated with urolithin $\mathrm{A}(100 \mathrm{nM})$ or cyclosporin $\mathrm{A}(2 \mathrm{mM})$ for 30 min prior to high glucose $(25 \mathrm{mM})$ treatment for $48 \mathrm{~h}$. Cyclosporin A was used as a desensitization control, $n=6$. $\mathbf{f}, \mathbf{g}$ Cells were pretreated with Ru360 $(1 \mu \mathrm{M})$ for 30 min prior to high glucose for $48 \mathrm{~h}$. f DCFDA staining was performed and the cells stained with DCF-DA was analyzed with flow cytometer, $n=4$. g MitoSOX staining was achieved and the MitoSOX-positive cells were counted by using flow cytometer. Antimycin A $(10 \mu \mathrm{M})$ was used for inducing mtROS accumulation to make a positive control for analysis, $n=4$. Quantitative data are shown as a mean \pm S.E.M. All flow cytometer figures are representative. $* p<0.05$. 
relatively weak effect of ellagic acid or other urolithins compared with urolithin A, we focused on the urolithin A's regulatory effect on neuronal cells under high glucose condition. Furthermore, high glucose condition for $24-48 \mathrm{~h}$ increased rhod-2-positive cell population in SH-SY5Y, which indicates high glucose induces mitochondrial calcium influx (Fig. 1c). We found that urolithin A pretreatment inhibited high glucose-stimulated mitochondrial calcium accumulation, but inhibited mitochondrial permeability transition pore (mPTP) opening (Fig. 1d, e). High glucoseinduced intracellular and mtROS levels were suppressed by pretreatment with the MCU (mitochondrial calcium uniporter) inhibitor Ru360 (Fig. 1f, g). These findings indicate that the effect of urolithin A on decreasing mitochondrial calcium accumulation is important for maintaining mtROS homeostasis in SH-SY5Y cells under high glucose.

\section{Effect of urolithin A on high glucose-induced amyloidogenesis and neuronal degeneration in neuronal cells and streptozotocin (STZ)-induced diabetic mice}

To determine the effect of urolithins on amyloidogenesis in neuronal cells under high glucose, we measured secreted $\mathrm{A} \beta$ (1-42) levels in SH-SY5Y and human-induced pluripotent stem cells (iPSC)-derived neuronal differentiated cells (iPSC-ND). We found that high glucose increased APP and BACE1 expressions in a time-dependent manner (Supplementary Fig. 2). In addition, we observed a significant decrease in $\mathrm{A} \beta$ (1-42) concentration levels in SH-SY5Y cells incubated in medium containing urolithin $\mathrm{A}$ and urolithin $\mathrm{B}$, but not ellagic acid, urolithin $\mathrm{C}$, or urolithin $\mathrm{D}$ (Fig. 2a). Since the inhibitory effect of urolithin $A$ on $A \beta$ secretion was the most potent compared with other substrates, we tested the effect of urolithin $A$ on $A \beta$ concentration in iPSC-ND cells under high glucose. In iPSCND-conditioned medium, the $\mathrm{A} \beta(1-42)$ concentration level in the urolithin A pretreatment with high glucose group was lower than that in the vehicle pretreatment with high glucose group (Fig. 2b). Urolithin A pretreatment suppressed high glucose-induced APP and BACE1 expression in both SH-SY5Y and iPSC-ND cells (Fig. 2c, d). High glucoseinduced APP and BACE1 expression levels were suppressed by Ru360 or mtROS-specific scavenger MitoTEMPO pretreatment (Fig. 2e, f). These results suggest that urolithin $\mathrm{A}$-inhibited $\mathrm{A} \beta$ production is mediated by the suppression of mitochondrial calcium and mtROS accumulation. Furthermore, we determined the effect of urolithin A on STZ-induced cognitive impairment, amyloidogenesis, Tau phosphorylation, and $\mathrm{A} \beta$ deposition in the prefrontal cortex and hippocampus of mouse brain tissues. Urolithin A did not affect body weight gain in either control or STZ mice (Fig. 2g). Blood glucose levels in urolithin A-injected control mice were significantly lower than that in vehicle-injected control mice, but this difference between vehicle-injected STZ and urolithin A-injected STZ mice was not statistically significant (Fig. 2h). For evaluating cognitive function, we performed a Y-maze spontaneous alternation test. We observed that STZ injection decreased the spontaneous alternation rate, which was reversed by urolithin A injection (Fig. 2i). In the prefrontal cortex and hippocampus tissues, urolithin $\mathrm{A}$ injection inhibited STZ-induced expression of APP, BACE1, and pTau (S262 and S396) and the level of A $\beta$ (1-42) (Fig. 2j, k). Therefore, our in vitro and in vivo experimental findings suggest that urolithin A treatment can inhibit amyloidogenesis and neuronal degeneration in DM models.

\section{Role of transglutaminase type 2 (TGM2) in urolithin A reduced mitochondria-ER contacts, mitochondrial calcium influx, mtROS accumulation, and amyloidogenesis under high glucose}

We investigated the effect of high glucose on the mRNA expression of proteins that regulate mitochondrial calcium influx, such as VDAC1, MCU1, mitochondrial calcium uptake 1 (MICU1), mitochondrial calcium uptake 2 (MICU2), MCU regulator 1 (MCUR1), and MCU-dominant negative beta subunit $(M C U B)$. We found that the mRNA expression levels of $V D A C 1$ and $M C U 1$ were upregulated in SH-SY5Y under a high glucose condition (Fig. 3a). However, urolithin A pretreatment did not affect high glucoseinduced VDAC1 and MCU1 mRNA and protein expression (Fig. 3b, c). In addition, Ru360 pretreatment did not change the high glucose-stimulated VDAC1 and MCU1 protein expressions (Supplementary Fig. 3). We further investigated the effect of high glucose and urolithin A on the interactions between the ER and mitochondria. As shown in Fig. 3d, we found that high glucose increased the co-localization of MitoTracker-positive fluorescence with ER-Trackerpositive fluorescence in SH-SY5Y cells, which was reversed by urolithin A pretreatment. In coimmunoprecipitation and in situ proximity ligation assay (PLA) experiments, high glucose increased the direct interaction between IP3R1, IP3R3, and VDAC1, which was abolished by urolithin A pretreatment (Fig. 3e, f). In addition, we investigated the mRNA expression changes of mitochondria-ER contacts regulatory adapter proteins, such as Bcl-2-associated X protein $(B A X), \mathrm{Bcl}-2$-like1 (BCL2L1), B-cell lymphoma 2 (BCL2), glucose-regulated protein 75 (GRP75), and TGM2. However, high glucose only stimulated TGM2 mRNA expression, which was reversed by urolithin A pretreatment (Fig. 3g) [20-22, 37]. This TGM2 induction by high glucose was suppressed by MitoTEMPO pretreatment (Supplementary Fig. 4). We confirmed the inhibitory effect of urolithin A pretreatment on high 
A

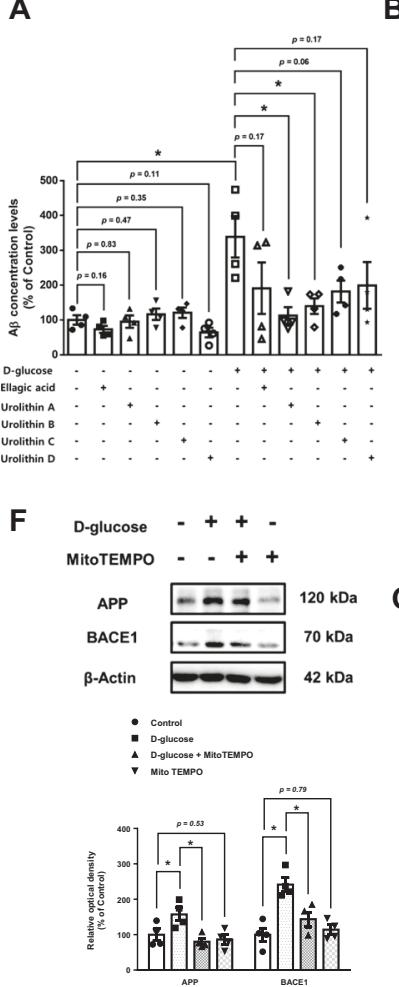

B
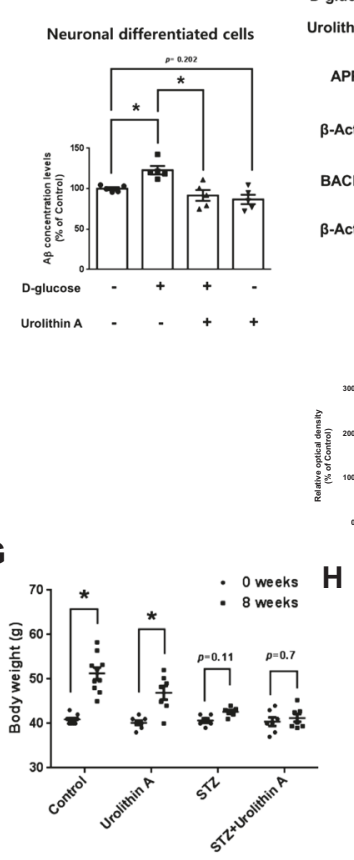

C

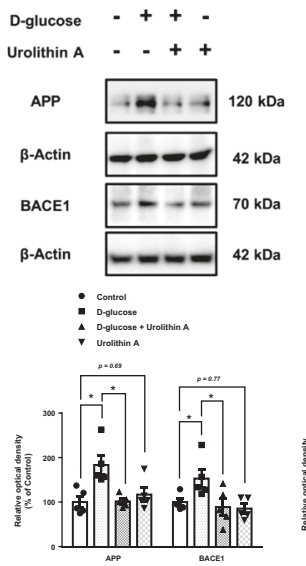

D

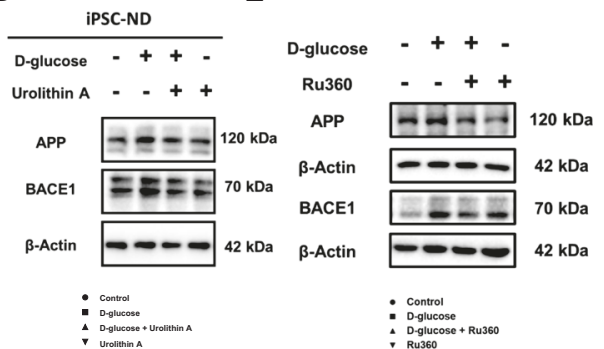

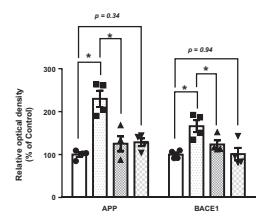

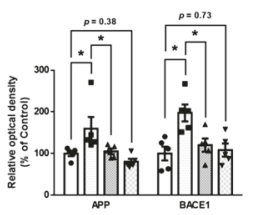

I

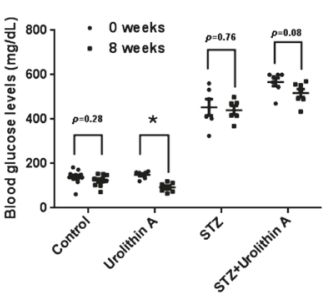

I

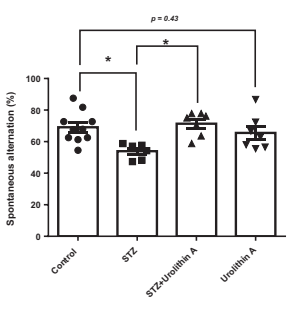

$\mathbf{J}$
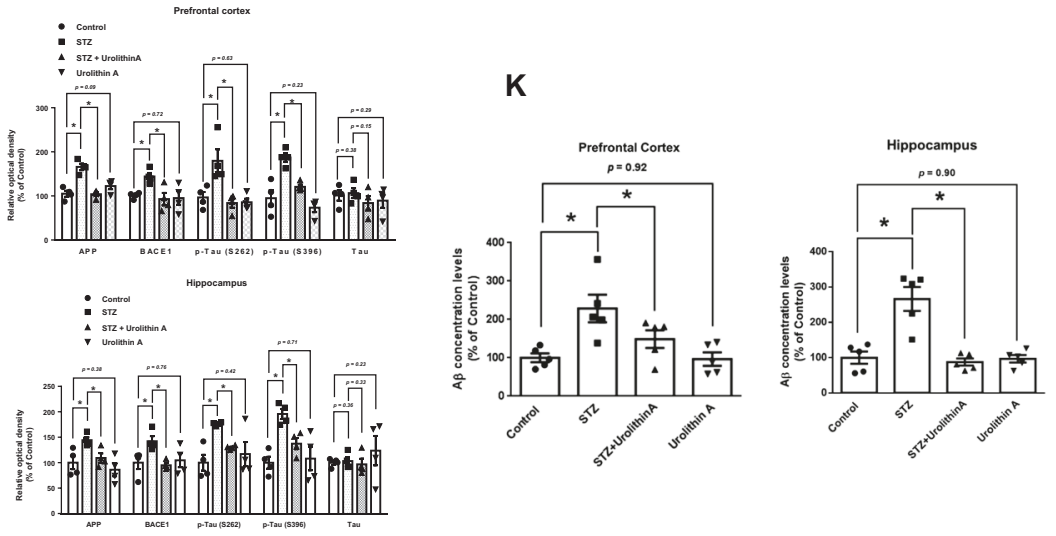

Fig. 2 Effect of urolithin $A$ on amyloidogenesis in neuronal cells under high glucose condition. a SH-SY5Y cells were treated with $100 \mathrm{nM}$ of ellagic acid, urolithin A, B, C, and D for $30 \mathrm{~min}$ prior to high glucose exposure ( $25 \mathrm{mM}$ of D-glucose) for $48 \mathrm{~h}$. Amyloid beta (A $\beta$ ) concentration in culture medium was calculated by using highsensitive A $\beta$ ELISA, $n=4$. b iPSC-ND cells were treated with urolithin $\mathrm{A}(100 \mathrm{nM})$ for $30 \mathrm{~min}$ prior to high glucose exposure for $48 \mathrm{~h}$. $\mathrm{A} \beta$ concentration in media supernatant was determined by highsensitive $A \beta$ ELISA kit. $A \beta$ concentration levels of each group were presented as percentage of control, $n=5$. c, d APP and BACE1 protein expressions were detected by western blot analysis. c SHSY5Y and $\mathbf{d}$ iPSC-ND cells were exposed to urolithin A for $30 \mathrm{~min}$ prior to high glucose treatment for $24 \mathrm{~h}, n=4-5$. e, f SH-SY5Y cells were treated with Ru360 $(1 \mu \mathrm{M})$ or MitoTEMPO ${ }^{\mathrm{TM}}(1 \mu \mathrm{M})$ for $30 \mathrm{~min}$ prior to high glucose treatment for $24 \mathrm{~h}$. APP and BACE1 protein expressions were detected by western blot analysis. $\beta$-Actin was used as a loading control, $n=4-5$. $\mathbf{g}$-i Normal and STZ-induced diabetic

glucose-induced TGM2 expression in both SH-SY5Y and iPSC-ND cells (Fig. 3h, i). In addition to urolithin Ainhibited TGM2 expression, it also decreased high glucose- mice were delivered vehicle $(0.5 \mathrm{mM} \mathrm{NaOH})$ or urolithin A solution $(2.5 \mathrm{mg} / \mathrm{kg})$ via intraperitoneal injection for 8 weeks, $n=6-10$. g Body weight of the mice was measured at the timepoint of initial urolithin $\mathrm{A}$ injection ( 0 week) and final injection ( 8 weeks). h Blood glucose levels of the mice were evaluated in each experimental group. $\mathbf{i}$ The memory function of each group of mice was assessed with spontaneous alternation test (Y-maze). The percentage of spontaneous alternation of entering the other side of the arm and total duration time was analyzed. $\mathbf{j}$, $\mathbf{k}$ Brain tissue of mice were divided into the two part as prefrontal cortex (left) and hippocampus (right) at the termination of experiment. $\mathbf{j}$ The expression levels of APP, BACE1, and p-Tau (S262 and S396) were analyzed by western blot analysis, $n=4$. $\mathbf{k}$ A $\beta$ concentration levels of the mice brain tissue were measured with highsensitive $A \beta$ ELISA kit. Left panel is the result from the prefrontal cortex tissue, and right panel is the result from the hippocampus, $n=$ 5. Quantitative data are shown as a mean \pm S.E.M. $* p<0.05$.

induced TGM2 activity in SH-SY5Y cells (Fig. 3j). In mouse brain samples, urolithin A injection significantly decreased STZ-induced TGM2 expression in both the 


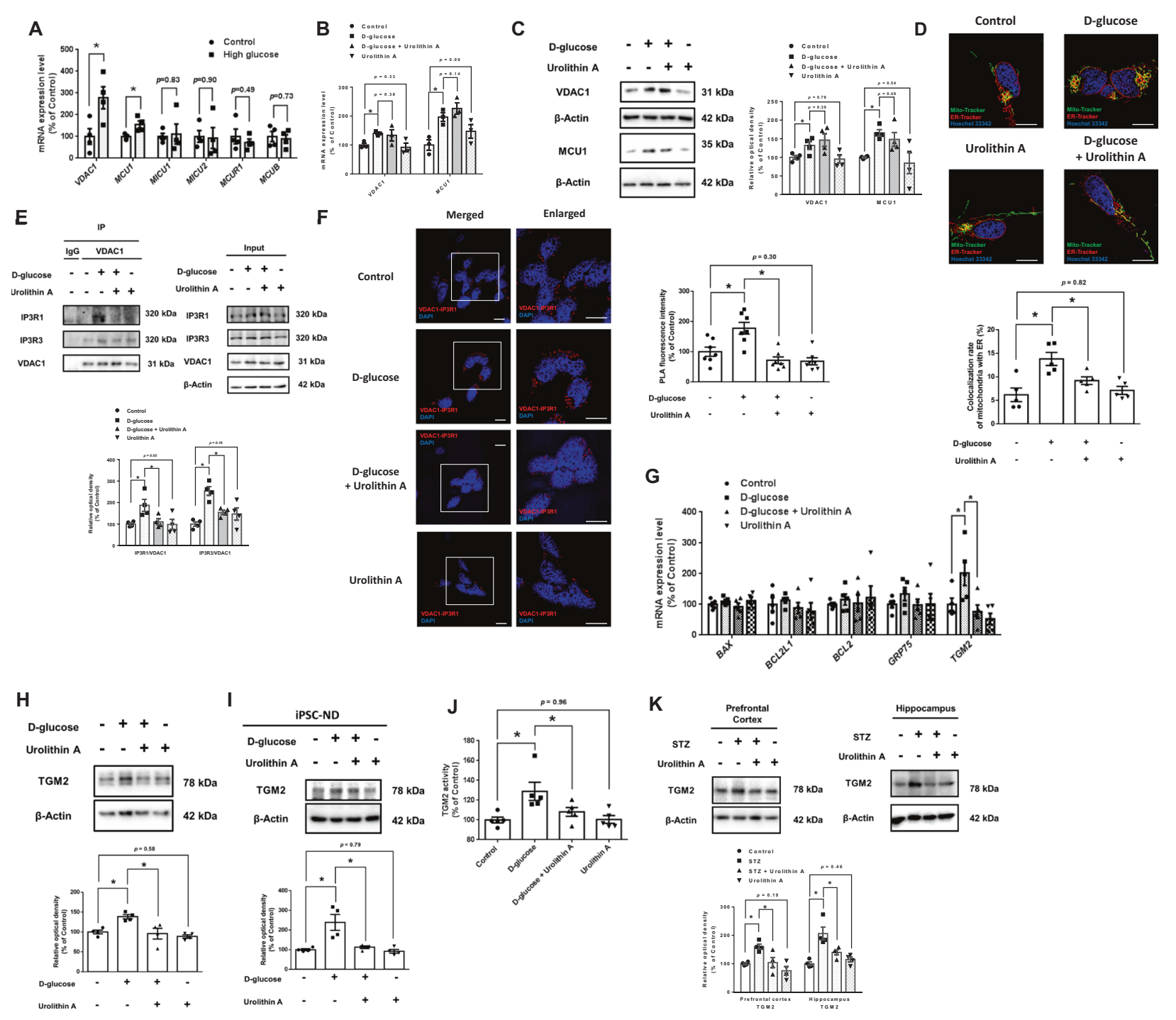

Fig. 3 Effect of urolithin A in MAM-regulated mitochondrial calcium under high glucose condition. a The mRNA expression levels of VDAC1, MCU1, MICU1, MICU2, MCUR1, and MCUB were analyzed in the SH-SY5Y cells treated with vehicle or high glucose for 24 h by using qPCR, $n=4$. b-i SH-SY5Y cells pretreated with urolithin A $(100 \mathrm{nM})$ for $30 \mathrm{~min}$ prior to high glucose exposure for $24 \mathrm{~h}$. b The mRNA expression levels of VDAC1 and MCU1 was quantified by qPCR, $n=3$. c Protein expression levels were analyzed by western blot by using anti-VDAC1 and anti-MCU1 antibodies, $n=4$. d Physical association of mitochondria and ER was visualized by the staining of MitoTracker (green), ER-tracker (red), and Hoechst 33342 (blue). Merged images are shown and representative. $n=5$. Magnification $\times 1,000$. Scale bars are $8 \mu \mathrm{m}$. e Co-immunoprecipitation of IP3R1 and IP3R3 with IgG or VDAC1 antibodies are shown in left panel. Total protein expression of IP3R1, IP3R3, VDAC1 and $\beta$-Actin are shown in right panel, $n=4$. f Interaction between VDAC1 and

prefrontal cortex and hippocampus tissues (Fig. 3k). Taken together, urolithin A inhibited TGM2 expression, which is critical for the suppression of IP3R1-VDAC1 interactions in neuronal cells exposed to a high glucose environment. To determine the role of TGM2 in mitochondria-ER contacts
IP3R1 (VDAC1-IP3R1, red) in SH-SY5Y cells was assessed by PLA assay. $n=6$. Magnification $\times 1,000$. Scale bars are $8 \mu \mathrm{m}$. $\mathbf{g}$ The mRNA expressions of $B A X, B C L 2 L 1, B C L 2, G R P 75$, and TGM2 in SH-SY5Y cells were analyzed by qPCR. Normalization was achieved by $18 \mathrm{~s}$ $r R N A$ expression levels, $n=5$. h TGM2 protein expression in $\mathrm{SH}-$ SY5Y cells was analyzed by western blot in cells treated urolithin A for $30 \mathrm{~min}$ prior to high glucose exposure for $24 \mathrm{~h}, n=4$. i TGM2 protein expression was analyzed in iPSC-ND treated with urolithin A $(100 \mathrm{nM})$ for $30 \mathrm{~min}$ prior to high glucose exposure for $24 \mathrm{~h}, n=4$. j TGM2 activity was evaluated in the SH-SY5Y cells treated urolithin A $(100 \mathrm{nM})$ for $30 \mathrm{~min}$ prior to high glucose exposure for $24 \mathrm{~h}$ with TGM2 activity assay kit, $n=5$. k Prefrontal cortex and hippocampus tissues were dissected from the STZ-induced diabetic mice treated with vehicle or urolithin A. TGM2 expression was analyzed by western blot analysis, $n=4$. Quantitative data are shown as a mean \pm S.E.M. $* p<0.05$.

and amyloidogenesis under high glucose, we investigated the effects of TGM2 silencing in SH-SY5Y cells. We observed that TGM2 silencing abolished the high glucoseincreased co-localization of MitoTracker-positive fluorescence and ER-Tracker-positive fluorescence in SH-SY5Y 


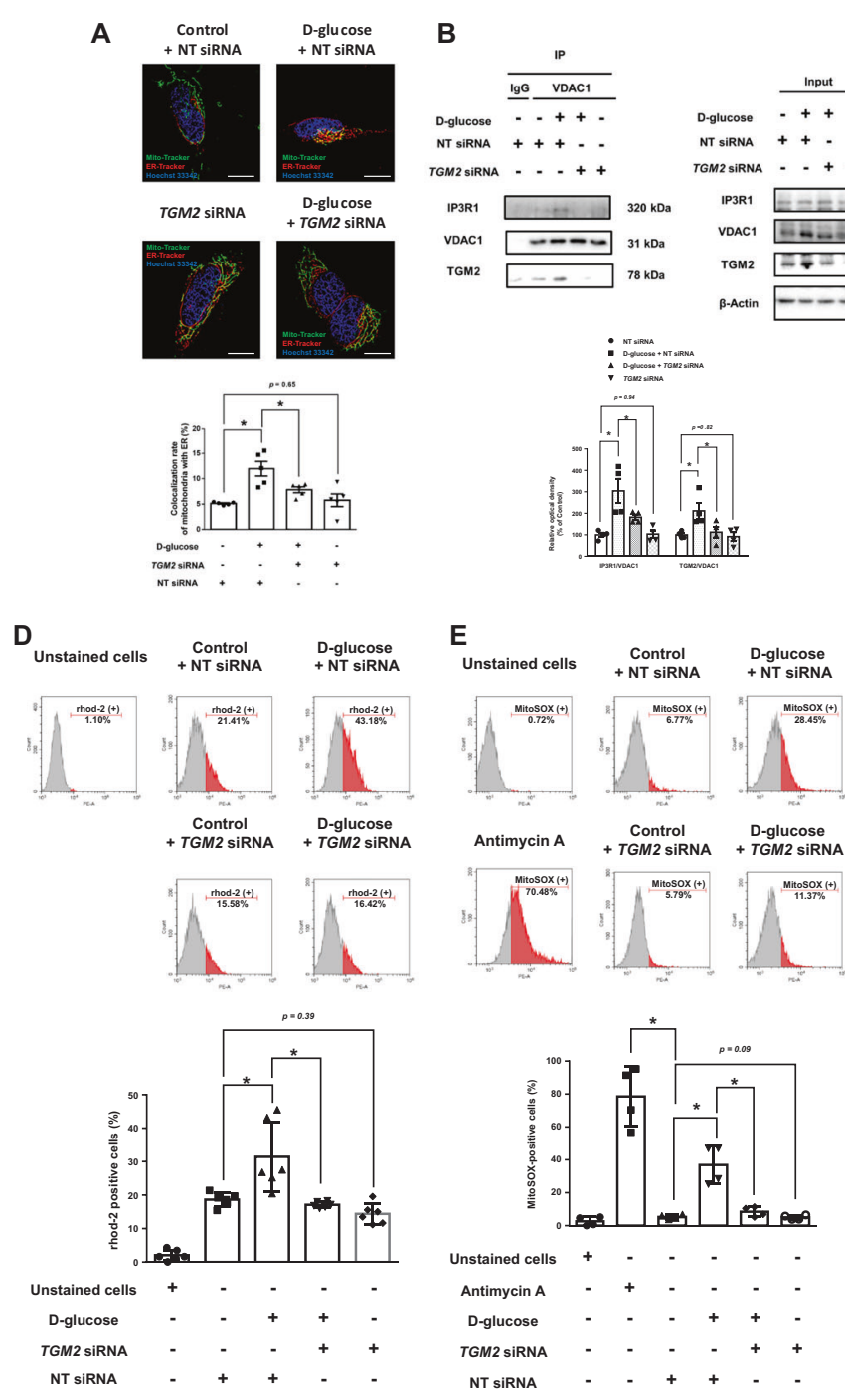

C
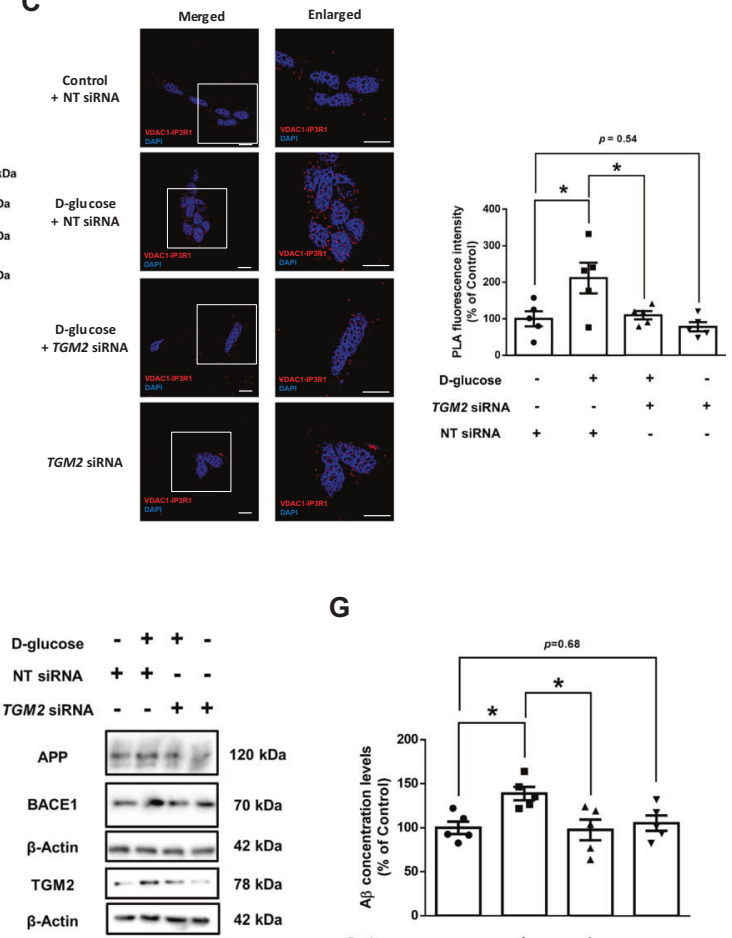

G

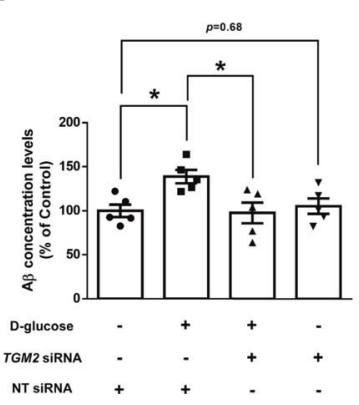

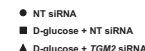

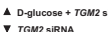

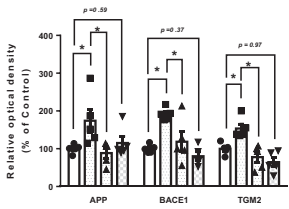

with rhod-2 fluorescent dye $(2 \mu \mathrm{M})$ for $20 \mathrm{~min}$, and rhod-2-positive cells were analyzed with flow cytometer. Unstained cells were used as a negative control, $n=6$. e The mtROS levels were evaluated with MitoSOX fluorescent dye $(1 \mu \mathrm{M})$ for 20 min MitoSOX-positive cells were analyzed with flow cytometer. Antimycin A $(10 \mu \mathrm{M})$ was used for inducing mtROS accumulation to make a positive control for analysis. All flow cytometer figures are representative, $n=4$. f APP, BACE1, and TGM2 protein expressions in SH-SY5Y cells were analyzed by western blot, $n=5$. $\mathrm{g}$ A $\beta$ concentration levels of the cell culture media originated from the cells transfected with TGM2 siRNA or NT siRNA prior to high glucose exposure for $48 \mathrm{~h}$ were measured with high-sensitive A $\beta$ ELISA kit, $n=5$. Quantitative data are shown as a mean \pm S.E.M. $* p<0.05$.

PLA assay, $n=5$. Magnification $\times 1,000$. Scale bars are $8 \mu n$ d Mitochondrial calcium levels were measured by staining the cells

cells (Fig. 4a). In co-immunoprecipitation and PLA experiments, TGM2 silencing downregulated high glucoseinduced IP3R1-VDAC1 interactions (Fig. 4b, c). High glucose-induced mitochondrial calcium and mtROS levels in SH-SY5Y cells were suppressed by TGM2 silencing (Fig. 4d, e). Furthermore, TGM2 silencing prevented high glucose-induced A $\beta$ secretion, as well as APP and BACE1 expression (Fig. 4f, g). Based on these findings, urolithin Anormalized high glucose-induced TGM2 expression hinders
IP3R1-VDAC1 interactions, which leads to the suppression of mitochondrial calcium influx and mtROS accumulation under high glucose.

\section{Role of urolithin A-mediated AIP-AhR complex disruption in high glucose-induced TGM2 expression}

We identified the mechanism of how urolithin A suppresses high glucose-induced TGM2 expression. We observed that 
the protein expression level of aryl hydrocarbon receptor (AhR) was increased in 24 and $48 \mathrm{~h}$ of high glucose treatment, but not AhR-interacting protein (AIP) was not increased (Fig. 5a). Then, we determined the effect of urolithin $\mathrm{A}$ on the mRNA expression of AhR regulatory adapter proteins, such as prostaglandin $\mathrm{E}$ synthase 3 (PTGES3), AIP, and heat shock protein $90 \alpha$ family class A member 1 (HSP90AA1), in SH-SY5Y cells under high glucose. However, high glucose and urolithin A did not affect their mRNA or protein expression of AIP in SHSY5Y cells (Supplementary Fig. 5A, B). We found that high glucose increased the nuclear AhR expression level, which was reversed by urolithin A pretreatment (Fig. 5b, c). High glucose-induced TGM2 mRNA and protein expression levels were inhibited by pretreatment with the AhR inhibitor CH-223191 (Fig. 5d, e). In addition, high glucose significantly inhibited the interaction between AhR and AIP, which was recovered by urolithin A pretreatment (Fig. 5f, g). These findings indicate that urolithin A inhibits high glucose-activated AhR signaling by disrupting the formation of the AIP-AhR complex to suppress TGM2 expression.

\section{Protective effects of urolithin A and TGM2 silencing on A $\beta$-stimulated mitochondrial calcium influx, mtROS accumulation, Tau phosphorylation, and neuronal cell death}

Next, we investigated the effect of urolithin $A$ on $A \beta$ stimulated mitochondrial calcium influx, mtROS accumulation, and neuronal cell death. $\mathrm{A} \beta$ treatment significantly increased the LDH release level in SH-SY5Y cells at 48 and $72 \mathrm{~h}$ (Fig. 6a). In SH-SY5Y and iPSC-ND cells, urolithin A pretreatment inhibited $\mathrm{A} \beta$-increased mitochondrial calcium levels (Fig. 6b, c). We confirmed that urolithin A pretreatment prevented $A \beta$-induced mtROS accumulation in SHSY5Y and iPSC-ND cells (Fig. 6d, e). Furthermore, urolithin A pretreatment prevented LDH release from SHSY5Y cells treated with $A \beta$ for $72 \mathrm{~h}$ (Fig. $6 \mathrm{f}$ ). We further investigated the effect of TGM2 silencing on $\mathrm{A} \beta$-treated neuronal cells and APP Swedish mutant SK-N-MC cells (APPSwe) secreting A $\beta$. TGM2 silencing decreased A $\beta$ reduced mitochondrial calcium levels in SH-SY5Y cells (Fig. 7a). TGM2 silencing or urolithin A pretreatment decreased $A \beta$-increased mitochondrial calcium levels in APPSwe cells (Fig. 7b). In addition, TGM2 silencing inhibited $A \beta$-induced mtROS accumulation in SH-SY5Y (Fig. 7c) and APPSwe cells transfected with TGM2 siRNA showed lower mtROS levels than NT siRNA transfected APPSwe cells (Fig. 7d). Urolithin A pretreatment also suppressed $A \beta$-induced mtROS accumulation in APPSwe cells (Fig. 7d). TGM2 silencing significantly dephosphorylated A $\beta$-stimulated Tau phosphorylation (Fig. 7e).
In APPSwe cells, both TGM2 silencing and urolithin A pretreatment decreased Tau phosphorylation (Fig. 7f). The LDH release levels in SH-SY5Y cells with $\mathrm{A} \beta$ treatment were also decreased by TGM2 silencing (Fig. $7 \mathrm{~g}$ ). Collectively, our data suggest that urolithin A pretreatment and TGM2 silencing prevents $\mathrm{A} \beta$-induced mitochondrial calcium influx, mtROS accumulation, Tau phosphorylation, and cell death in neuronal cells.

\section{Discussion}

We highlight the inhibitory effect of urolithin A on TGM2-mediated mitochondrial calcium influx, which alleviates high glucose-stimulated amyloidogenesis and neuronal degeneration. The production of $A \beta$ under high glucose conditions has been mainly focused on APP processing, but our study showed the relationship between mitochondrial calcium influx and amyloidogenesis under high glucose conditions [5]. In addition, we identified a potent metabolite that is found in the human gut microbiome. First, the present study revealed that nanomolar concentration of urolithin A $(100 \mathrm{nM})$ regulated mitochondrial calcium influx, mtROS accumulation, amyloidogenesis, and neuronal cell death under high glucose. It has been reported that urolithins circulate in blood plasma at a concentration in the range of $0.2-20 \mu \mathrm{M}$ after the ingestion of ellagic acid [25]. In an urolithin production study with two human fecal samples, the urolithin A production rate was shown to be dependent on individual fecal microflora composition [38]. Previous studies have shown that urolithin A treatment controlled cellular biological functions, including autophagy, inflammation, and apoptosis, which may be cell-type specific. Indeed, several researchers showed that sub-micromolar and micromolar concentrations of urolithin A stimulated autophagy, apoptosis, and cell cycle arrest in colorectal cancer cells $[39,40]$. Conversely, other researchers reported the antioxidative and protective effects of urolithin A treatment in neuronal cell lines [33, 34]. Furthermore, urolithin A treatment inhibited TNF $\alpha$ production and lipopolysaccharide-stimulated macrophage polarization $[29,41]$. These findings suggest that urolithin A has antioxidative and anti-inflammatory potential in neuronal cells. High glucose-induced protein glycation is associated with the progression of general complications in diabetes [42]. Also, it has been reported that urolithin A has an anti-glycation effect on neuronal cells [33]. However, O-GlcNAcylation inhibition by an O-GlcNAcase inhibitor does not affect high glucose-induced oxidative stress or mitochondrial dysfunction [43]. Considering that the O-GlcNAcylation of proteins is a major risk factor inducing mitochondrial dysfunction, those findings 
A

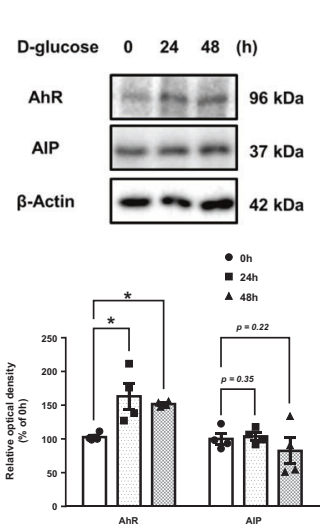

B
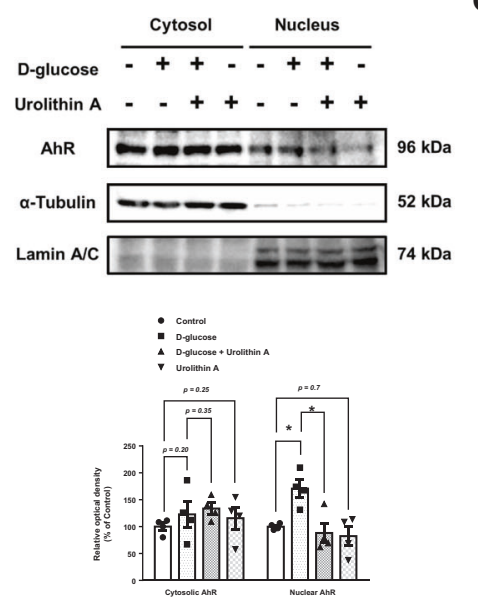

D

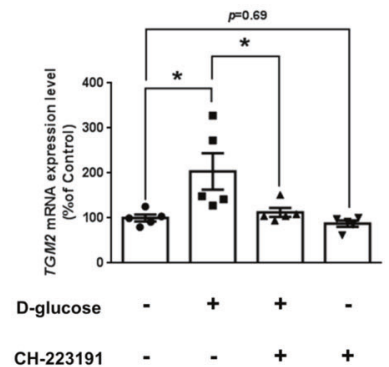

G

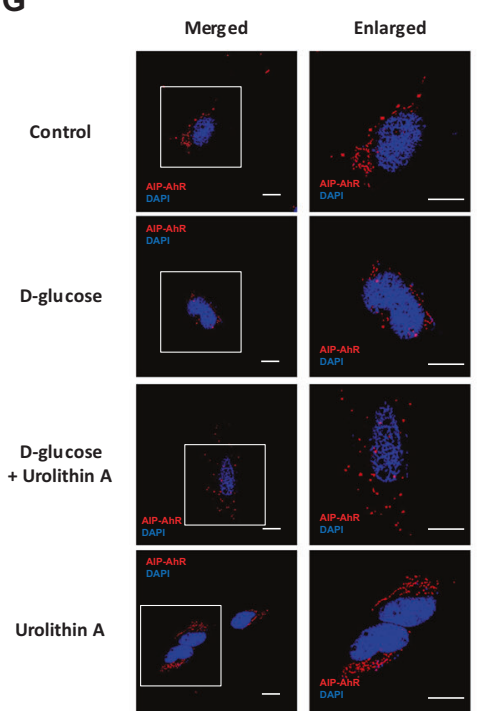

Fig. 5 Effect of urolithin A on inhibition of TGM2 expression via formation of AIP-AhR transcriptomal complex. a SH-SY5Y cells were treated with high glucose for 0,24 , and $48 \mathrm{~h}$. AhR and AIP expressions were analyzed by western blot analysis, $n=4$. b Cells treated with urolithin $\mathrm{A}$ ( $100 \mathrm{nM})$ for 30 min prior to high glucose ( 25 $\mathrm{mM}$ ) exposure for $24 \mathrm{~h}$. Subcellular fractions of cytosol and nucleus were analyzed with western blot analysis. AhR expression level was analyzed with anti-AhR antibody. The anti- $\alpha$-tubulin (cytosol) and -Lamin A/C (nucleus) antibodies were used for confirmation of fractionization, $n=4$. $\mathrm{c}$ Nuclear localization of AhR was confirmed in the cells treated with urolithin $\mathrm{A}(100 \mathrm{nM})$ for $30 \mathrm{~min}$ prior to high glucose $(25 \mathrm{mM})$ exposure for $24 \mathrm{~h}$. Cells stained with AhR (green) antibody and DAPI (blue) were visualized and merged images are shown and
C

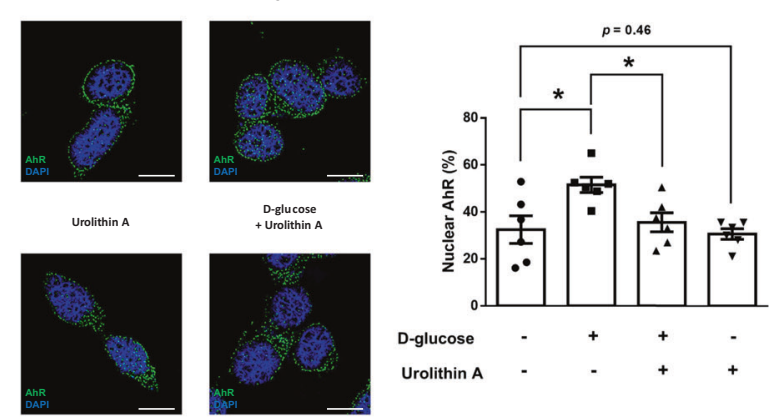

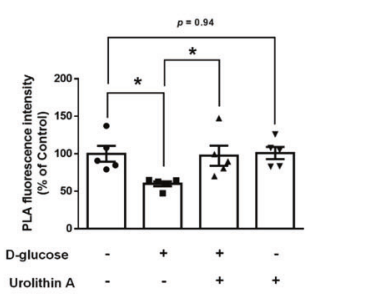

$\mathbf{F}$

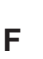

$\frac{\text { IP }}{\lg \text { G }}$

D-glucose $\frac{\text { Input }}{-++}$

Urolithin $A-\cdots+$

D-glucose -+++

Urolithin A - - + +

AIP

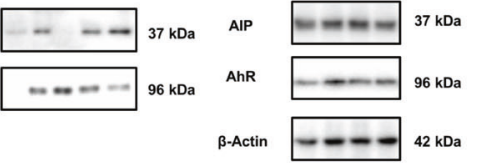

AIP / AhR

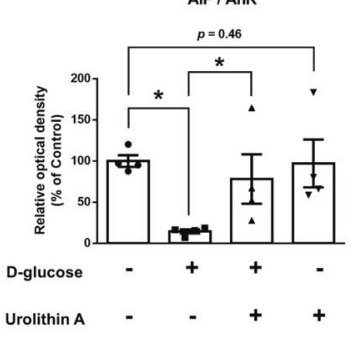

representative, $n=5$. Magnification $\times 1,000$. Scale bars are $8 \mu \mathrm{m}$. d, e Cells treated with $\mathrm{CH}-223191(10 \mu \mathrm{M})$ for $30 \mathrm{~min}$ prior to high glucose exposure for $24 \mathrm{~h}$. d TGM2 mRNA expression level was analyzed by using qPCR, $n=5$. e TGM2 protein expression level was analyzed by western blot. $\beta$-Actin was used as loading control, $n=4$. f, g Cells treated with urolithin A $(100 \mathrm{nM})$ for $30 \mathrm{~min}$ prior to high glucose $(25 \mathrm{mM})$ exposure for $24 \mathrm{~h}$. f Co-immunoprecipitation of AIP with anti-IgG or -AhR antibodies are shown in left panel. Total protein expression of AIP, AhR, and $\beta$-Actin are shown in right panel, $n=4$. g Interaction between AIP and AhR (AIP-AhR, red) in SH-SY5Y cells was assessed by PLA assay, $n=5$. Magnification $\times 1,000$. Scale bars are $8 \mu \mathrm{m}$. Quantitative data are shown as a mean \pm S.E.M. $* p<0.05$. 
A

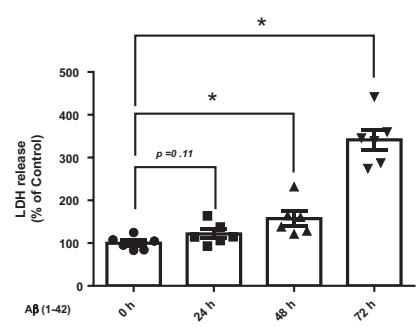

B
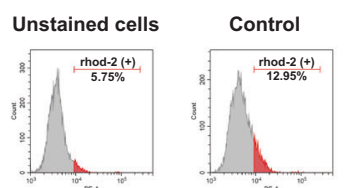

Ionomycin

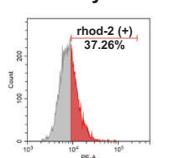

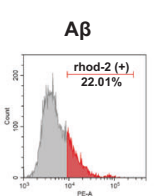

Urolithin A

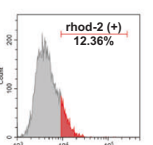

C

iPSC-ND

Unstained cells Control $\quad A \beta$

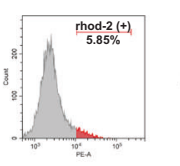

Ionomycin
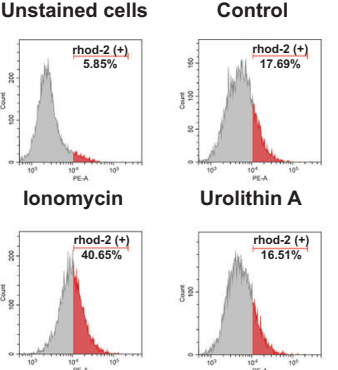

Urolithin A

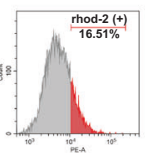

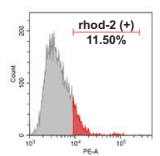

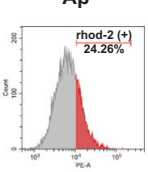

$A \beta+$ Urolithin $A$
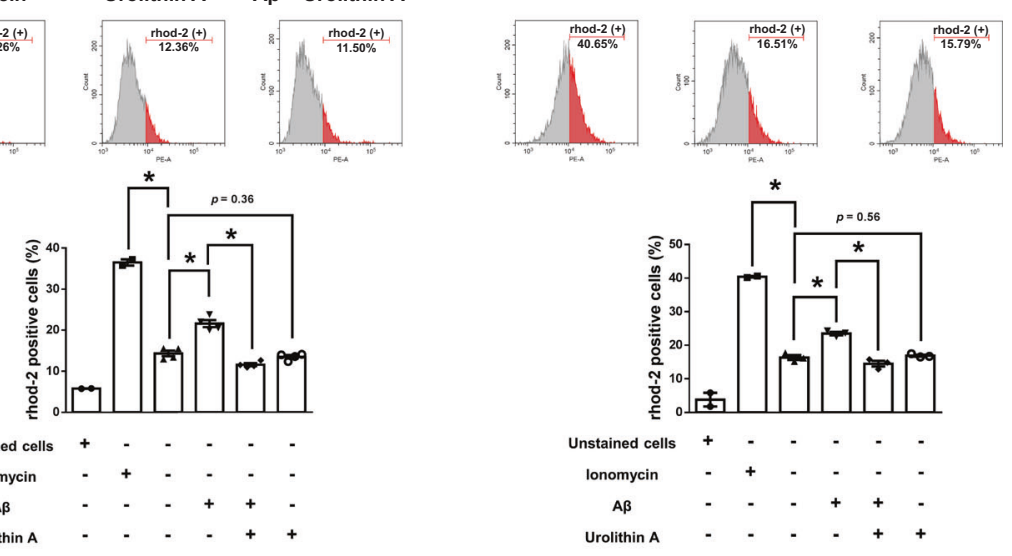

E IPSC-ND

Unstained cells Control $\quad A \beta$
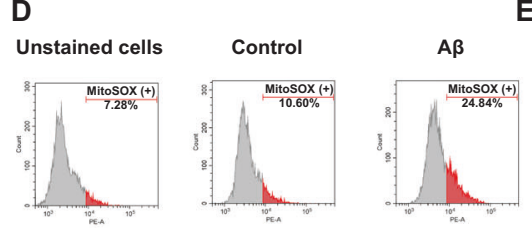

$A \beta+$ Urolithin $A$

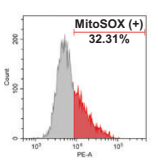

Urolithin A
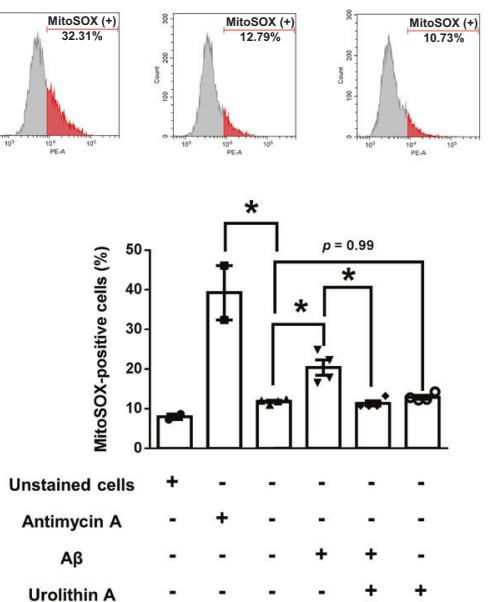

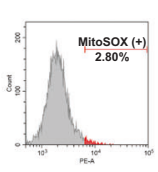

Antimycin A

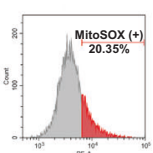

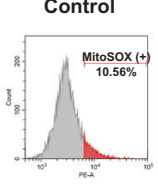

Urolithin A

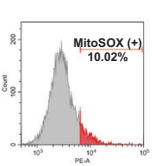

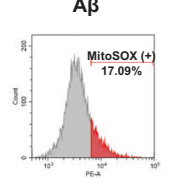

$A \beta+$ Urolithin $A$

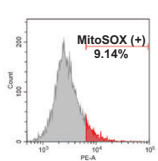

$\mathbf{F}$

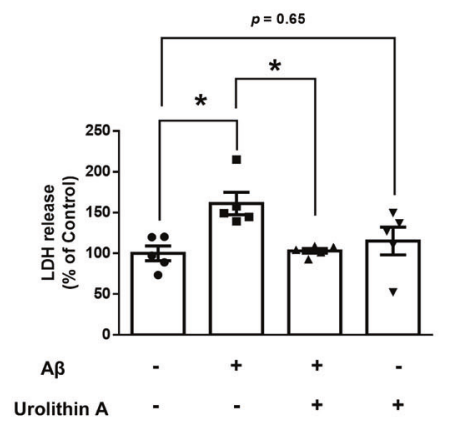

Fig. 6 Protective effects of urolithin A on A $\beta$-induced mitochondrial calcium influx, mtROS accumulation, and neuronal cell death. a LDH release assay was performed in the SH-SY5Y cells treated with Amyloid beta (A $\beta)(1-42)$ for 24,48 , and $72 \mathrm{~h}, n=6$. b SH-SY5Y cells treated with urolithin A $(100 \mathrm{nM})$ for 30 min prior to high glucose exposure for $24 \mathrm{~h}$. Mitochondrial calcium levels were measured by staining the cells with rhod-2 fluorescent dye $(2 \mu \mathrm{M})$ for $20 \mathrm{~min}$, and rhod-2-positive cells were analyzed with flow cytometer. Unstained cells were used as a negative control and ionomycin $(2 \mu \mathrm{M})$ treated cells were used for a positive control, $n=4$. $\mathbf{c}$ iPSC-ND treated with urolithin $\mathrm{A}(100 \mathrm{nM})$ for $30 \mathrm{~min}$ prior to high glucose exposure for $24 \mathrm{~h}$. rhod- 2 stained cells were measured by flow cytometer, $n=4$. d, e MitoSOX-positive SH-SY5Y and iPSC-ND cells were analyzed with flow cytometer. Antimycin A $(10 \mu \mathrm{M})$ was used for inducing mtROS accumulation to make a positive control for analysis, $n=4$. All flow cytometer figures are representative. $\mathbf{f} \mathrm{LDH}$ release assay was performed in the SH-SY5Y cells treated with urolithin A for $30 \mathrm{~min}$ prior to A $\beta(1-42)$ for $72 \mathrm{~h}, n=5$. Quantitative data are shown as a mean \pm S.E.M. $* p<0.05$.

The present study demonstrates that high glucose increases the mRNA and protein expression of VDAC1 and MCU1, but urolithin A pretreatment does not alter their suggest that urolithin A-inhibited protein glycation is not associated with anti-oxidative stress in neuronal cells under high glucose [44]. 
A

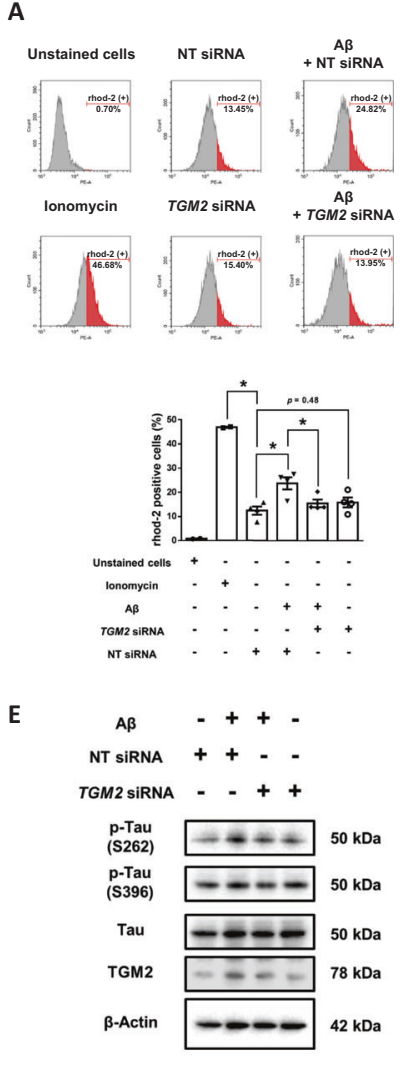

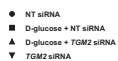

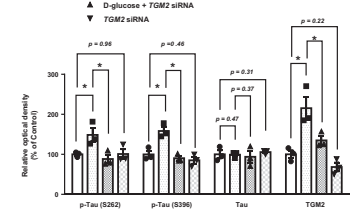

APPSW

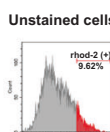

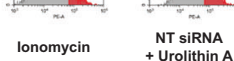

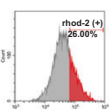
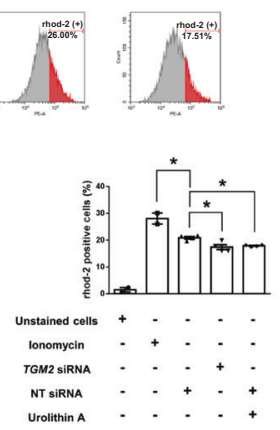

$\mathbf{F}$

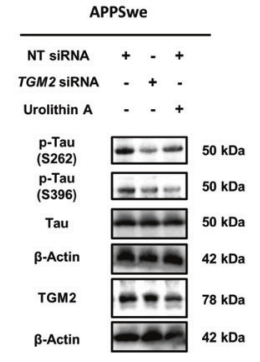

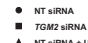

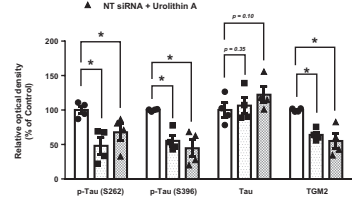

C

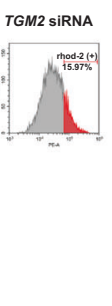

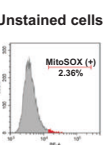

Antimycin A

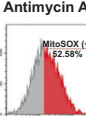

$\sqrt{120.50 \times(t)}$
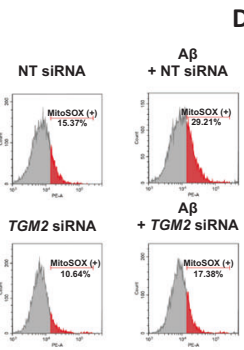

D

APPSW
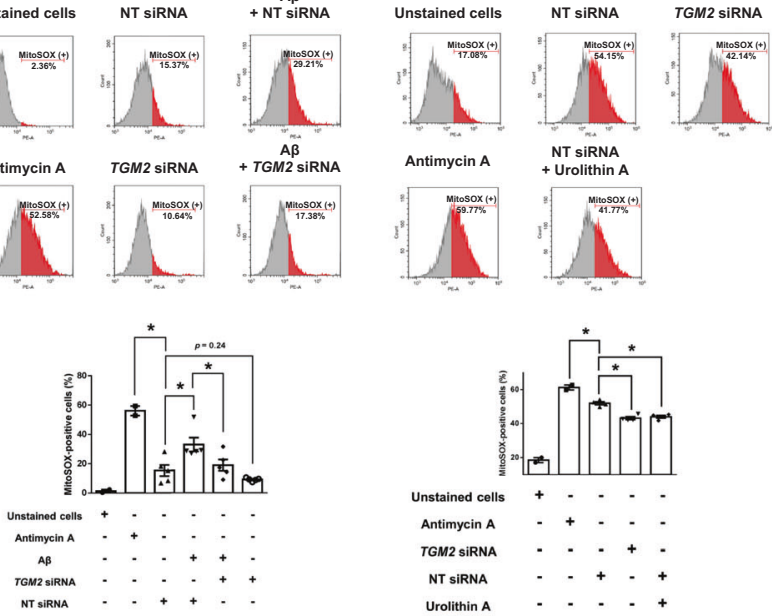

G
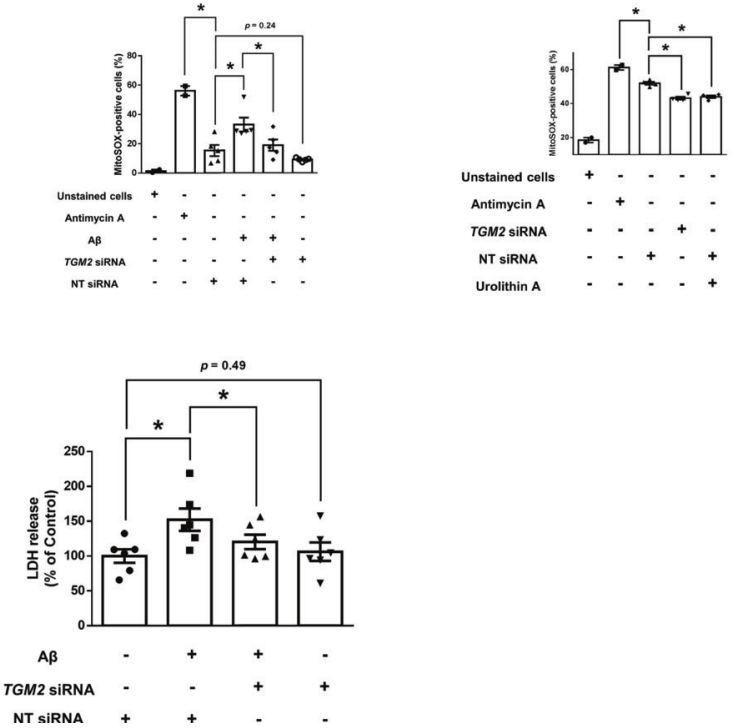

Fig. 7 Role of TGM2 in A $\beta$-induced mitochondrial calcium overload, mtROS accumulation, tau phosphorylation, and neuronal cell death. a-d SH-SY5Y cells were transfected with TGM2 siRNA or NT siRNA prior to A $\beta$ exposure for $48 \mathrm{~h}$. APPSwe cells were transfected with TGM2 siRNA or NT siRNA and then cells were treated urolithin A for $48 \mathrm{~h}$. a, b rhod-2-positive cells were analyzed by flow cytometer, $n=4$. c, $\mathbf{d}$ MitoSOX-positive cells were quantified by flow cytometer, $n=4-5$. e Tau phosphorylation levels at S262 and S396

expression in SH-SY5Y cells. Consistent with our results, increased mPTP, mitochondrial calcium influx from the ER in a high glucose environment, and DM models have been well documented [23, 24, 45]. Previous researchers reported that high glucose conditions stimulated the expression of VDAC1, MCU1, and MCUR1, which are critical for mitochondrial calcium influx, migration, and apoptosis of vascular endothelial cells and T2D islets [13, 24]. Conversely, it has been reported that MCU plays a key role in mitochondrial calcium influx and mtROS accumulation, although high glucose treatment did not affect MCU expression in HepG2 cells [23]. Other researchers found that MICU1 downregulation by Sp1 signaling contributed to diabetic cardiomyopathy in $\mathrm{db} / \mathrm{db}$ mice [46]. Taken were investigated in the SH-SY5Y cells transfected with TGM2 siRNA or NT siRNA prior to A $\beta$ exposure for $24 \mathrm{~h}, n=3$. f Tau phosphorylation levels at S262 and S396 in the APPSwe cells transfected with $T G M 2$ siRNA or NT siRNA prior to exposure urolithin A for $24 \mathrm{~h}$, $n=3$. $\mathbf{g}$ LDH release in the cells transfected with TGM2 siRNA or NT siRNA prior to $\mathrm{A} \beta(1-42)$ for $72 \mathrm{~h}$ was investigated, $n=6$. Quantitative data are shown as a mean \pm S.E.M. $* p<0.05$.

together, the present and previous findings indicate that the effect of high glucose on the expression of mitochondrial calcium influx regulatory proteins is cell-type specific. Several studies demonstrated the intracellular calcium regulatory potential of urolithins in various types of cells. Urolithin A-activated miR-10a-5p decreased Orail mRNA expression, which inhibited intracellular calcium entry in murine $\mathrm{CD}^{+}$cells [47]. In addition, urolithin $\mathrm{C}$ activated L-type calcium channel opening for glucose-stimulated insulin secretion [48]. We first showed a suppressive effect of urolithin A on mitochondrial calcium influx from the ER involving mtROS accumulation in neuronal cells under high glucose. However, we found that urolithin A did not affect the expression of mitochondrial calcium influx regulating 
proteins, such as VDAC1, MCU1, MICU1, MICU2, MCUR1, and MCUB, in neuronal cells under high glucose. Indeed, GRP75 antagonist pretreatment or TGM2 deletion significantly prevented mitochondrial calcium overload without the changes of VDAC1 and MCU1 expressions $[20,22]$. Based upon previous and present findings, we suggest that urolithin A-reduced mitochondrial calcium influx is independent on high glucose-increased VDAC1 and MCU1 expression levels. Furthermore, we demonstrated that urolithin A-suppressed mitochondrial calcium influx alleviated high glucose-stimulated $\mathrm{A} \beta$ production by downregulating APP and BACE1 expression. Also, our in vivo data showed that urolithin A alleviated the expression of APP and BACE1, cognitive impairment, Tau phosphorylation, and $\mathrm{A} \beta$ deposition in STZ-injected mice. Consistent with our data, a previous study demonstrated that urolithin A reduced neuronal apoptosis, $\mathrm{A} \beta$ deposition, and cognitive impairment in an APPSwe/PS1 mouse model, suggesting that urolithin $\mathrm{A}$ is a promising therapeutic $\mathrm{AD}$ drug candidate [49]. In addition, we found that urolithin A decreased blood glucose levels in control mice but not in STZ-injected mice. A previous comparative study investigating the gut microbiota in metabolic syndrome patients reported an inverse correlation between urolithin $\mathrm{A}$ and glucose levels [50]. Also, it was reported that urolithin A injection for 12 weeks increased the insulin sensitivity in high fat diet-ingested C57BL/6 mice [51]. Therefore, the urolithin A-suppressed blood glucose levels in control mice but not in STZ-injected mice are likely due to insulin sensitivity.

To the best of our knowledge, there has been no study on the regulatory effect of urolithin A on TGM2-mediated mitochondrial calcium influx. Therefore, we demonstrate for the first time that TGM2 plays a critical role in urolithin A-induced mitochondria-ER contacts disruption in neuronal cells under high glucose. TGM2 is a multifunctional transamidating acyltransferase that catalyzes calciumdependent posttranslational modifications inducing protein cross-linking, glutamine deamination, polyamines incorporation, and guanosine triphosphate hydrolysis [52-55]. Recently, Manuela D'Eleto et al. reported that TGM2 plays a key role in assembling the IP3R-GRP75-VDAC1 complex, which leads to MAM protein profile conversion and calcium influx from the ER to mitochondria [22]. Downregulation of the interaction between TGM2 and GRP75 decreased the number of ER-mitochondria contract sites and mitochondrial calcium influx through an increase of the interaction between IP3R and GRP75 [22]. TGM2 knockout cell line exhibited decreased mitochondrial calcium uptake level, which indicates that reduction of TGM2 expression without regulation of GRP75 expression is sufficient for mitochondrial calcium influx from ER [22]. Although urolithin A does not alter the expression level of GRP75, these findings suggest that urolithin A-inhibited TGM2 expression decreases the interaction between GRP75 and IP3R leading to an impairment of the mitochondrial calcium influx from ER. In addition, there are several reports showing the physiological role of hyper-activated TGM2 in DM-associated complications related to the small resistance arteries, and kidneys [56, 57]. Consistently, our data also showed the upregulated expression of TGM2 in high glucose-treated neuronal cells, as well as in the hippocampus and prefrontal cortex of STZ-injected mice, which were abolished by urolithin A injection. Therefore, those findings suggest that TGM2 may be a promising therapeutic target for the prevention and treatment of hyperglycemiarelated $\mathrm{AD}$.

We showed that urolithin A inhibited TGM2 expression through high glucose-activated AhR signaling both in vitro and in vivo. Consistent with our data, a previous study reported that high glucose conditions stimulated the transcriptional activation of AhR through the formation of the Egr-1/AP-2/AhR complex [58]. In addition, it was documented that urolithin $\mathrm{A}$ acted as an $\mathrm{AhR}$ antagonist. A previous competitive binding assay study suggested that urolithin A competed with indole to directly bind to AhR [59]. These findings suggest a possibility that urolithin A plays a regulatory role in high glucose-induced TGM2 expression. However, other previous reports have shown an inhibitory role of AhR in TGM2 expression. For example, the upregulation of TGM2 activity was reported in liver tissues of AhR null mice, and treatment with an AhR activator 2,3,7,8-tetrachlorodibenzo-p-dioxin inhibited TGM2 expression in malignant human keratinocytes [60]. Furthermore, treatment with urolithin A or the synthetic urolithin A analog urolithin AS03 stimulates AhR expression and enhances the barrier function of the gut by inducing the expression of tight junction regulatory proteins, such as claudin 4 and occludin [61]. Because there has been no report identifying the role of $\mathrm{AhR}$ in urolithin Aregulated TGM2 expression in neuronal cells, additional studies will be needed to determine the distinctive effect of urolithin A on TGM2 expression in various types of cells, including neuronal cells. Furthermore, we demonstrated that inhibiting the interaction between AIP and AhR under high glucose was critical for TGM2 expression, which was reversed by urolithin A pretreatment. Previous researchers showed that AIP stimulated AIP-AhR-HSP90 complex formation, which led to the cytosolic localization of AhR by inhibiting the interaction of AhR with importin $\beta$ [62-64]. Therefore, AIP-AhR interaction is the key for the regulation of TGM2 expression by urolithin A in neuronal cells under high glucose.

We also showed the inhibitory effect of urolithin A on the A $\beta$-induced mtROS accumulation promoted by TGM2 expression. A recent study provided evidence of the 
upregulation of mitochondria-ER contacts in live hippocampal neurons from APP transgenic rats coupled with impaired mitochondrial functions [65]. The increased number of mitochondria-ER contacts in $\mathrm{AD}$ models is associated with the accumulation of $\mathrm{A} \beta$ plaques and neurofibrillary tangles $[16,66]$. Also, impaired mitochondrial calcium efflux precedes disease pathogenesis in a 3xTG-AD mouse model [17]. In addition, $A \beta$ oligomers increased mitochondrial calcium influx from the ER in neurons [67]. According to previous reports, TGM2 stimulated the formation of insoluble toxic amyloid aggregates, which exacerbated the pathogenesis of $\mathrm{AD}$ [68]. A significant increase in TGM2-induced $\varepsilon$-( $\gamma$-glutamyl) lysine bonds in paired helical filament Tau were observed in the postmortem brains of AD patients [69]. Consistent with these findings, our data also showed TGM2 upregulation by $\mathrm{A} \beta$ treatment in SH-SY5Y cells. Furthermore, we demonstrated that TGM2 silencing or urolithin A treatment significantly prevented $A \beta$-induced mitochondrial calcium influx, mtROS accumulation, Tau phosphorylation, and apoptosis of neuronal cells, suggesting that urolithin A-mediated TGM2 suppression is a promising therapeutic strategy for $\mathrm{AD}$ patients.

In conclusion, we demonstrated that urolithin A inhibited AhR-mediated TGM2 expression, suppressing mitochondrial calcium influx through the disruption of mitochondria-ER contacts, which alleviated mtROS accumulation and amyloidogenesis in neuronal cells under high glucose (Fig. 8). To the best of our knowledge, the present study is the first to identify urolithin A-mediated mitochondrial calcium regulatory effects on high glucoseinduced $\mathrm{A} \beta$ production and neuronal degeneration. Furthermore, we show that TGM2 plays a key role in urolithin A-regulated mitochondrial calcium influx and demonstrate a detailed mechanism of the inhibition of high glucosestimulated TGM2 expression by urolithin A treatment. Therefore, our findings provide new insights into the urolithin A-based therapeutic strategy for the prevention and treatment of DM-associated AD pathogenesis.

\section{Materials and methods}

\section{Materials}

The neuroblastoma cell line SH-SY5Y was purchased from the Korean Cell Line Bank (Seoul, Korea). The SK-N-MC neuroblastoma cell line stably expressing the Swedish mutation (K595N/M596L) of the amyloid precursor protein (APPSwe) was kindly provided by Dr. KI Park (Yonsei University, Seoul, Korea). Fetal bovine serum (FBS) was purchased from Hyclone (Logan, UT, USA), respectively. Urolithin A (SML1791), urolithin B (SML1649), ellagic

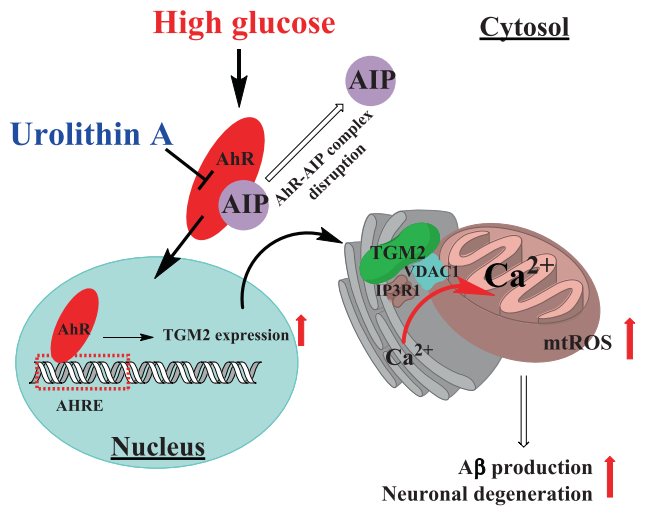

Fig. 8 The schematic model for action mechanism of urolithin A on high glucose-induced neuronal amyloidogenesis by modulating TGM2-dependent ER-mitochondria contacts and calcium influx. High glucose induces neuronal degeneration and amyloidogenesis via mitochondrial calcium influx and mtROS accumulation. High glucose disrupts AhR-AIP complex and MAM formation. Urolithin A inhibits AhR nuclear translocation under high glucose exposure. Urolithin A reduces high glucose-induced AhR-mediated transcription of TGM2, which is critical for IP3R1-VDAC1 interactions. Conclusively, urolithin A-mediated suppression of TGM2 expression prevents the neuronal degeneration and $\mathrm{A} \beta$ production under high glucose conditions.

acid (E2250), D-glucose (G7021), hydrogen peroxide $\left(\mathrm{H}_{2} \mathrm{O}_{2}\right)$, carbonyl cyanide m-chlorophenyl hydrazone (CCCP, C2759), Ru360 (557440), antimycin A, CH223191 (C8124), streptozotocin (S0130), and ionomycin (I0634) were obtained from Sigma-Aldrich (St. Louis, USA). Urolithin C (U847015) and urolithin D (U847020) were purchased from Toronto research chemicals (North York, ON, Canada); a lyophilized powder of amyloid beta $\left(\mathrm{A} \beta_{1-42}\right)$ was purchased from Lugen Sci (Bucheon, Korea); and $\mathrm{A} \beta$ oligomerization was performed according to a previous report [70]. The PCR primers for VDAC1, MCU1, MICU1, MICU2, MCUR1, MCUB, BAX, BCL2L1, BCL2, GRP75, TGM2, PTGES3, AIP, HSP90AA1, and 18s rRNA were synthesized by Bioneer (Daejeon, Korea). Small interfering RNAs (siRNAs) for TGM2 and a non-targeting (NT) siRNA were purchased from Bioneer. IP3R (ab5804), APP (ab32136) and BACE1 (ab2077) antibodies were obtained from Abcam (Cambridge, UK), p-Tau S396 (sc101815), Tau (sc-390476), VDAC1 (sc-390996), $\alpha$-tubulin (sc-32293), lamin A/C (sc-20681), and $\beta$-Actin (sc-47778) antibodies were acquired from Santa Cruz (CA, USA). MCU1 (D2Z3B), TGM2 (D11A6), and AhR (D5S6H) antibodies were bought from Cell Signaling Technology (MA, USA). IP3R3 (NBP1-21399) and AIP (NB100-127) were delivered from Novus Biologicals (CO, USA). p-Tau S262 (44-750G) antibody was acquired from Thermo Fisher (Waltham, MA, USA). 4',6-Diamidino-2-Phenylindole, Dihydrochloride (DAPI) (D1306) was also 
purchased from Thermo Fisher. To avoid the oxidation of chemicals, all materials were prepared fresh at the moment of use. All reagents were of the highest purity available.

\section{Cell culture}

SH-SY5Y cells were cultured in high glucose Dulbecco's Modified Eagle Medium (DMEM) provided by Thermo Fisher, which was supplemented with a $1 \%$ penicillin-streptomycin solution (Gibco, Grand Island, NY, USA) and 10\% FBS (Hyclone, Logan, UT, USA). APPSwe SK-N-MC cells were cultured in high glucose DMEM containing a $1 \%$ penicillin-streptomycin solution and $10 \%$ FBS. Cells were grown in $60 \mathrm{~mm}$ dishes, $100 \mathrm{~mm}$ culture dishes, or a 96-well plate (Corning, NY, USA) in an incubator $\left(37^{\circ} \mathrm{C}, \mathrm{CO}_{2} 5 \%\right.$, and air $\left.95 \%\right)$. When the cells reached $80 \%$ confluency, the culture medium was replaced with serum-free low-glucose DMEM for $24 \mathrm{~h}$ for starvation. After incubation, the cells were incubated in serum-free low-glucose DMEM supplemented with the indicated agents for the designated treatment time. To generate a high glucose condition, confluent cells were treated with $25 \mathrm{mM}$ D-glucose.

\section{Neuronal differentiation from iPSCs}

The iPSCs were obtained from Kangstem Biotech (Seoul, Korea). iPSCs were cultured on recombinant human vitronectin (rhVTN, A14700, Thermo Fisher)-coated plates. For neuronal stem cell induction, we used PSC neural induction medium (A1647801, Thermo Fisher). After neuronal differentiation from iPSCs, we plated neural stem cells (NSCs) on laminin- (23017, Thermo Fisher) and poly-L-ornithine (Sigma, P3655)-coated dishes. For neural differentiation, NSCs were cultured in Neurobasal medium (21103, Thermo Fisher) with the serum-free supplement B27 (17504, Thermo Fisher) and GlutaMax (35050, Thermo Fisher) for 10 days. To enhance neural differentiation, dibutyryl-cAMP (D0627, Sigma) was added to the neural differentiation medium between days 7 and 10 .

\section{LDH cytotoxicity assay}

Cell concentration was optimized following the protocol provided in the LDH release assay kit (EZ-LDH, DoGenBio, Seoul, Korea, DG-LDH500). SH-SY5Y cells were then seeded at a density of $1 \times 10^{4}$ cells/well in a 96well plate. The media was replaced with serum-free lowglucose DMEM once the cells reached 90\% confluency. After centrifugation of the plate at $600 \times g$, the supernatant was collected and incubated with the LDH assay mixture at room temperature (RT) for $30 \mathrm{~min}$. The $\mathrm{LDH}$ release level was analyzed by measuring the optical density at $450 \mathrm{~nm}$ with an Epoch 2 spectrophotometer (BioTek, VT, USA).

\section{Water soluble tetrazolium salt (WST-1) cell viability assay}

The viability of SH-SY5Y cells were measured with the WST-1 cell viability assay (EZ-Cytox ${ }^{\text {TM }}$; Daeil Labservice, Seoul, Korea, \# EZ-1000). Cells were cultured in 96-well plates until $80 \%$ confluency, and then, the growth media was changed with serum-free low-glucose DMEM. Urolithin A $(100 \mathrm{nM})$ or hydrogen peroxide $\left(\mathrm{H}_{2} \mathrm{O}_{2} ; 100 \mu \mathrm{M}\right)$ was added to the cells for $30 \mathrm{~min}$ before D-glucose treatment for $72 \mathrm{~h}$. Cells were incubated in $10 \mu \mathrm{l}$ of EZ-Cytox ${ }^{\mathrm{TM}}$ reagent in $100 \mu \mathrm{l}$ of DMEM for $30 \mathrm{~min}$ at $37^{\circ} \mathrm{C}$. The absorbance at $450 \mathrm{~nm}$ was measured with an Epoch 2 spectrophotometer.

\section{Measurements of mtROS and calcium and the mitochondrial permeability transition pore assay}

MitoSOX Red (Thermo Fisher, M36008) and rhod-2 (Thermo Fisher, R1244) were used to determine the level of intracellular ROS, mtROS, and mitochondrial calcium. The level of MPTP opening was analyzed by using Calcein AM (Thermo Fisher, C1430) to determine cell death by measuring mPTP opening. $\mathrm{CoCl}_{2}(400 \mu \mathrm{M})$ was added to quench the cytosolic calcium. Each experiment was performed using a Cytoflex flow cytometer (Beckman Coulter, FL, USA), and the results were obtained by comparing the percentage of cells with high fluorescence intensity.

\section{Experimental design of the animal study}

The protocol for our animal study was approved by the Institutional Animal Care and Use Committee of Seoul National University (SNU-190801-4-1). Nine-week-old male CrljOri:CD1(ICR) mice were delivered from OrientBio (Korea). After measuring the weight of each mouse, they were housed for 3 days in controlled specific pathogenfree conditions of $22{ }^{\circ} \mathrm{C}, 70 \%$ relative humidity, a $12 \mathrm{~h}$ light: dark cycle, and had unlimited access to a normal diet and water. Animals were assigned to control and experimental groups through single-blind randomized controlled trial to eliminate bias. STZ was dissolved in $0.05 \mathrm{M}$ citrate buffer, and mice received intraperitoneal injections of STZ $(75 \mathrm{mg} / \mathrm{kg} /$ day $)$ or citrate buffer $(0.05 \mathrm{M})$ in a total volume of $200 \mu \mathrm{l}$ for 3 days. All mice were given $10 \%$ sucrose water for 7 days to limit the possibility of low-glucose shock in STZ-injected mice. Blood was taken from the tail, and blood glucose levels were determined 7 days after the STZ injection by using a blood glucose meter (Accu-Chek, Roche Diagnostics, Indianapolis, IN). Urolithin A was 
dissolved in $0.5 \mathrm{mM} \mathrm{NaOH}$ in PBS. Daily intraperitoneal injections of urolithin A $(2.5 \mathrm{mg} / \mathrm{kg} /$ day $)$ or $0.5 \mathrm{mM} \mathrm{NaOH}$ in a $200 \mu \mathrm{l}$ volume were administered for 8 weeks. Mice were monitored twice a day during all experiments. After 8 weeks of urolithin A or vehicle treatment, the body weight of each mouse was measured, and the blood glucose level in tail blood was measured.

\section{Y-maze spontaneous alternation test}

STZ-induced diabetic mice and normal mice were injected with urolithin A $(2.5 \mathrm{mg} / \mathrm{kg} /$ day $)$ or vehicle for 8 weeks. Before the Y-maze spontaneous alternation test, the mice were accommodated in the testing room for $3 \mathrm{~h}$ to reduce the effects of external environmental stimuli or unintentional stress on their behavior. First, mice were positioned in the Y-maze apparatus (Samjung, Seoul, Korea). Next, the mice were given the chance to explore the Y-maze for 8 $\mathrm{min}$, and the movements of the mice were recorded by using a video camera. The number of section accesses and triads were counted to evaluate the percentage of alternations, especially, it was counted just in case of all limbs were within the arm field of Y-maze. The alternation number represents the total alternations divided by the number of triads.

\section{Transglutaminase 2 activity measurement}

Transglutaminase (TGM) 2 activity assay kit was purchased from Biovision (Mountain View, CA, USA, K571). All procedure was followed by the supplier's protocol. TGM2 activity assay kit applies the production of a hydroxamate product from its deamidation activity. The hydroxamate finally formed a purple complex with stop solution and optical absorbance was measured at $525 \mathrm{~nm}$ with microplate reader. TGM2 activity was normalized by total protein of cell lysate sample.

\section{Small interfering RNA (siRNA) transfection}

SH-SY5Y cells were incubated with $25 \mathrm{nM}$ of the indicated siRNAs and the transfection reagent TurboFect (Thermo Fisher, R0531) for $24 \mathrm{~h}$ without antibiotics. The medium was changed to serum-free low-glucose DMEM. The sequences for the siRNAs used in this study are described in Supplementary Table S1. We confirmed that the siRNA efficacies by real-time qPCR for TGM2 were at least $70 \%$. A NT siRNA was used as a control.

\section{Real-time quantitative PCR}

Cells were treated with high glucose or a vehicle for $24 \mathrm{~h}$. Cells were then washed with PBS twice and lysed with buffer RL containing a 50X Dithiothreitol solution. Total RNA was extracted using an RNA Extraction kit (Takara, Japan, 9767) according to the manufacturer's instructions. Reverse-transcription PCR was conducted with $1 \mu \mathrm{g}$ of total RNA using a Maxime RT premix kit (iNtRON, Sungnam, Korea, 25081). The cDNA was amplified using a Maxime PCR PreMix Kit (iNtRON, 25165) and a MyGenie 96 (Bioneer, Daejeon, Korea). The relative mRNA expression level of the target gene was analyzed using a Rotor-Gene 6000 device (Corbett Research, Cambridge, UK) with the TB Green Premix Ex Taq (TaKaRa, RR420A). The specificity, efficiency, and fidelity of PCR primers for real-time quantitative PCR were validated by checking PCR products and analyzing the melting curves. Primer sequences are listed in Supplementary Table S2. The relative mRNA expression levels of VDAC1, MCU1, MICU1, MICU2, MCUR1, MCUB, BAX, BCL2L1, BCL2, GRP75, TGM2, PTGES3, $A I P$, and HSP90AAl were analyzed by the delta-delta $\mathrm{Ct}$ method. The $18 s$ rRNA was used as a reference gene for data normalization.

\section{Western blotting and subcellular fractionation}

Protein concentrations were determined by bicinchoninic acid (BCA) Protein Assay kits (Pierce, Rockford, IL, USA, 23225). Sample proteins were resolved by SDS-PAGE gel electrophoresis and transferred to PVDF membranes. The membranes were incubated with the primary antibody at $4{ }^{\circ} \mathrm{C}$ overnight. The specific bands were visualized by the ChemiDoc XRS + System (Bio-Rad, Richmond, CA, USA). Subcellular fractionation was conducted to isolate cytosolic and nuclear proteins. Cells were cultured in 100 $\mathrm{mm}$ dishes and treated with the indicated reagents. For the preparation of cytosolic- and nuclear-fractionated samples, the EzSubcell subcellular fractionation/extraction kit (Atto, Tokyo, Japan, WSE-7421) was used. Cytosolic and nuclear samples for western blot analysis were prepared according to the manufacturer's instructions; $\alpha$-tubulin and lamin A/C were used as cytosolic and nuclear protein markers, respectively.

\section{Enzyme-linked immunosorbent assay (ELISA) for $A \beta$ secretion}

For the quantification of $A \beta(1-42)$ in cell culture medium, the A $\beta 42$ Human ELISA (Thermo Fisher, KHB3544) was achieved according to the supplier's protocols. SH-SY5Y cells were cultured to grow until $80 \%$ confluency. Supernatants were collected and ELISA was conducted. Mice brain tissue samples of prefrontal cortex and hippocampus were collected and lysed with RIPA buffer (ATTO, Tokyo, Japan). The A $\beta 42$ Mouse ELISA (Thermo Fisher, 
KMB3441) was performed according to the supplier's instruction.

\section{Immunocytochemistry}

For immunocytochemistry, SH-SY5Y cells were fixed with $4 \%$ paraformaldehyde for $10 \mathrm{~min}$ and then incubated in $0.5 \%$ Tween-20 for $10 \mathrm{~min}$. Cells were incubated with primary antibodies in PBS containing $0.1 \%$ Tween-20 (PBST; 1:100 dilution) for $2 \mathrm{~h}$ and washed with PBS three times. Cells were incubated with Alexa Fluor 488 or 555conjugated secondary antibodies in PBST (1:100 dilution) for $1 \mathrm{~h}$. Immunofluorescently stained samples were visualized by a super-resolution radial fluctuation (SRRF) imaging system (Andor Technology, Belfast, UK). Relative fluorescence intensities of AhR/DAPI were quantified with ImageJ software. For analyzing ER-mitochondrial contacts, cells were treated with chemicals or transfected with TGM2 siRNAs, as indicated in the figure legends, and incubated for $24 \mathrm{~h}$. After washing the cells with PBS three times, cells were incubated with MitoTracker green $(200 \mathrm{nM})$ and ER-Tracker $(200 \mathrm{nM})$ in serum-free medium for $20 \mathrm{~min}$ at $37^{\circ} \mathrm{C}$, and nuclei were stained with Hoechst 33342 (Thermo Fisher, 33342).

\section{In situ proximity ligation assay}

VDAC1/IP3R1 interactions were detected in situ using Duolink II secondary antibodies and detection kits (SigmaAldrich, DUO92001, DUO92005, and DUO92008) according to the supplier's protocols. Cells were fixed, and PLA probe anti-VDAC1 and anti-IP3R1 antibodies were applied. Then, secondary antibodies were added. If the antibodies were in close proximity $(<40 \mathrm{~nm})$, they ligated together. Polymerization and amplification solutions were supplemented to amplify the signal (red) of the closed circle and were visualized by SRRF microscopy. DAPI was used to counterstain the nuclei. Fluorescence images of negative control groups, such as no VDAC1 (IP3R1), no IP3R1 (VDAC1), and VDAC1-PACS2, were presented in the supplementary information (Supplementary Fig. 6).

\section{Co-immunoprecipitation}

SH-SY5Y cells transfected with NT or TGM2 siRNA were treated with vehicle or D-glucose $(25 \mathrm{mM})$ for $24 \mathrm{~h}$ and then lysed with co-immunoprecipitation lysis buffer $(20 \mathrm{mM}$ Tris-HCl pH 8.0, $137 \mathrm{mM} \mathrm{NaCl}, 1 \%$ Nonidet P-40, and 2 mM EDTA) containing a protease inhibitor cocktail for 30 min on ice. The concentration of protein in each lysate was determined by a BCA quantification assay (Thermo Fisher, 23225). VDAC1 or rabbit IgG antibodies were immobilized with protein $\mathrm{G}$ magnetic beads (Sure Beads, Bio-Rad, CA, USA, 161-4021). The immobilized magnetic beads were incubated with cell lysates for $6 \mathrm{~h}$ at $4{ }^{\circ} \mathrm{C}$. Washed beads were eluted with $20 \mathrm{mM}$ glycine buffer $(\mathrm{pH} 2.0)$ for $5 \mathrm{~min}$ and neutralized with $1 \mathrm{M}$ phosphate buffer and laemmli sample buffer. Then, the protein samples were boiled at $100^{\circ} \mathrm{C}$ for $5 \mathrm{~min}$.

\section{Statistical analysis}

All quantitative data were presented as the mean \pm standard error of the mean. Data were analyzed using SigmaPlot 12 software. The sample sizes for animal studies were determined by SigmaPlot 12 software. Comparisons between two experimental groups were performed using a two-tailed Student's $t$ test. The means of multiple experimental groups were compared using a one-way ANOVA, followed by the Student-Newman-Keuls test for multiple comparisons. A $p$ value of $<0.05$ was considered statistically significant.

Acknowledgements This research was supported by National R\&D Program through the National Research Foundation of Korea (NRF) funded by the Ministry of Science, ICT \& Future Planning (NRF2020R1A2B5B02002442, NRF-2018R1D1A1B07050212) and BK21 PLUS Program for Creative Veterinary Science Research.

Author contributions HJL: Conception and design, collection and assembly of data, data analysis and interpretation, and manuscript writing. YHJ: Conception and design, collection and assembly of data, data analysis and interpretation, and manuscript writing. GEC, JSK, CWC, JRL, SYK, and JHY: Collection of data. SJL: Data analysis and interpretation, manuscript writing. HJH: Conception and design, data analysis and interpretation, and manuscript writing.

\section{Compliance with ethical standards}

Conflict of interest The authors declare that they have no conflict of interest.

Publisher's note Springer Nature remains neutral with regard to jurisdictional claims in published maps and institutional affiliations.

\section{References}

1. Nagai N, Ito Y, Sasaki H. Hyperglycemia enhances the production of amyloid $\beta 1-42$ in the lenses of otsuka long-evans tokushima fatty rats, a model of human type 2 diabetes. Invest Ophthalmol Vis Sci. 2016;57:1408-17.

2. Lee HJ, Seo HI, Cha HY, Yang YJ, Kwon SH, Yang SJ. Diabetes and Alzheimer's disease: mechanisms and nutritional aspects. Clin Nutr Res. 2018;7:229-40.

3. Yang $\mathrm{Y}, \mathrm{Wu} \mathrm{Y}$, Zhang $\mathrm{S}$, Song W. High glucose promotes $\mathrm{A} \beta$ production by inhibiting APP degradation. PLoS ONE. 2013;8: e69824.

4. Macauley SL, Stanley M, Caesar EE, Yamada SA, Raichle ME, Perez R, et al. Hyperglycemia modulates extracellular amyloid- $\beta$ concentrations and neuronal activity in vivo. $\mathrm{J}$ Clin Invest. $2015 ; 125: 2463-7$. 
5. Lee HJ, Ryu JM, Jung YH, Lee SJ, Kim JY, Lee SH, et al. High glucose upregulates BACE1-mediated $\mathrm{A} \beta$ production through ROS-dependent HIF- $1 \alpha$ and LXR $\alpha /$ ABCA1-regulated lipid raft reorganization in SK-N-MC cells. Sci Rep. 2016;6:36746.

6. McConlogue L, Buttini M, Anderson JP, Brigham EF, Chen KS, Freedman SB, et al. Partial reduction of BACE1 has dramatic effects on Alzheimer plaque and synaptic pathology in APP Transgenic Mice. J Biol Chem. 2007;282:26326-34.

7. Ohno M, Sametsky EA, Younkin LH, Oakley H, Younkin SG, Citron M, et al. BACE1 deficiency rescues memory deficits and cholinergic dysfunction in a mouse model of Alzheimer's disease. Neuron. 2004;41:27-33.

8. Kumar P, Raman T, Swain MM, Mishra R, Pal A. Hyperglycemia-induced oxidative-nitrosative stress induces inflammation and neurodegeneration via augmented tuberous sclerosis complex-2 (TSC-2) activation in neuronal cells. Mol Neurobiol. 2017;54:238-54.

9. Akter K, Lanza EA, Martin SA, Myronyuk N, Rua M, Raffa RB. Diabetes mellitus and Alzheimer's disease: shared pathology and treatment? Br J Clin Pharm. 2011;71:365-76.

10. Cheignon C, Tomas M, Bonnefont-Rousselot D, Faller P, Hureau C, Collin F. Oxidative stress and the amyloid $\beta$ peptide in Alzheimer's disease. Redox Biol. 2018;14:450-64.

11. Onphachanh X, Lee HJ, Lim JR, Jung YH, Kim JS, Chae CW, et al. Enhancement of high glucose-induced PINK1 expression by melatonin stimulates neuronal cell survival: involvement of MT2 /Akt/NF-кB pathway. J Pineal Res. 2017;63:e12427.

12. Tomlinson DR, Gardiner NJ. Glucose neurotoxicity. Nat Rev Neurosci. 2008;9:36-45.

13. Chen W, Yang J, Chen S, Xiang H, Liu H, Lin D, et al. Importance of mitochondrial calcium uniporter in high glucose-induced endothelial cell dysfunction. Diab Vasc Dis Res. 2017;14:494-501.

14. Liu ZJ, Zhao W, Lei HY, Xu HL, Lai LY, Xu R, et al. High glucose enhances bupivacaine-induced neurotoxicity via MCUmediated oxidative stress in SH-SY5Y cells. Oxid Med Cell Longev. 2019;2019:7192798.

15. Vance JE. MAM (mitochondria-associated membranes) in mammalian cells: lipids and beyond. Biochim Biophys Acta. 2014;1841:595-609.

16. Pinho CM, Teixeira PF, Glaser E. Mitochondrial import and degradation of amyloid- $\beta$ peptide. Biochim Biophys Acta. 2014;1837:1069-74.

17. Jadiya P, Kolmetzky DW, Tomar D, Di Meco A, Lombardi AA, Lambert JP, et al. Impaired mitochondrial calcium efflux contributes to disease progression in models of Alzheimer's disease. Nat Commun. 2019;10:3885.

18. Area-Gomez E, Del Carmen Lara Castillo M, Tambini MD, Guardia-Laguarta C, de Groof AJ, Madra M, et al. Upregulated function of mitochondria-associated ER membranes in Alzheimer disease. EMBO J. 2012;31:4106-23.

19. Hedskog L, Pinho CM, Filadi R, Ronnback A, Hertwig L, Wiehager $\mathrm{B}$, et al. Modulation of the endoplasmic reticulummitochondria interface in Alzheimer's disease and related models. Proc Natl Acad Sci USA. 2013;110:7916-21.

20. Xu H, Guan N, Ren YL, Wei QJ, Tao YH, Yang GS, et al. IP3RGrp75-VDAC1-MCU calcium regulation axis antagonists protect podocytes from apoptosis and decrease proteinuria in an Adriamycin nephropathy rat model. BMC Nephrol. 2018;19:140.

21. Camara AKS, Zhou Y, Wen PC, Tajkhorshid E, Kwok WM. Mitochondrial VDAC1: a key gatekeeper as potential therapeutic target. Front Physiol. 2017;8:460.

22. D'Eletto M, Rossin F, Occhigrossi L, Farrace MG, Faccenda D, Desai $R$, et al. Transglutaminase type 2 regulates ERmitochondria contact sites by interacting with GRP75. Cell Rep. 2018;25:3573-81.e4.
23. Panahi G, Pasalar P, Zare M, Rizzuto R, Meshkani R. MCUknockdown attenuates high glucose-induced inflammation through regulating MAPKs/NF- $\mathrm{\kappa B}$ pathways and ROS production in HepG2 cells. PLoS ONE. 2018;13:e0196580.

24. Zhang E, Mohammed Al-Amily I, Mohammed S, Luan C, Asplund $\mathrm{O}$, Ahmed $\mathrm{M}$, et al. Preserving insulin secretion in diabetes by inhibiting VDAC1 overexpression and surface translocation in $\beta$ cells. Cell Metab. 2019;29:64-77.e66.

25. Espin JC, Larrosa M, Garcia-Conesa MT, Tomas-Barberan F. Biological significance of urolithins, the gut microbial ellagic acid-derived metabolites: the evidence so far. Evid Based Complement Altern Med. 2013;2013:270418.

26. Garcia-Villalba R, Beltran D, Espin JC, Selma MV, TomasBarberan FA. Time course production of urolithins from ellagic acid by human gut microbiota. J Agric Food Chem. 2013;61:8797-806.

27. Selma MV, Beltran D, Luna MC, Romo-Vaquero M, GarciaVillalba R, Mira A, et al. Isolation of human intestinal bacteria capable of producing the bioactive metabolite isourolithin A from ellagic acid. Front Microbiol. 2017;8:1521.

28. Selma MV, Tomas-Barberan FA, Beltran D, Garcia-Villalba R, Espin JC. Gordonibacter urolithinfaciens sp. nov., a urolithinproducing bacterium isolated from the human gut. Int J Syst Evol Microbiol. 2014;64(Pt 7):2346-52.

29. Piwowarski JP, Granica S, Zwierzynska M, Stefanska J, Schopohl $\mathrm{P}$, Melzig MF, et al. Role of human gut microbiota metabolism in the anti-inflammatory effect of traditionally used ellagitannin-rich plant materials. J Ethnopharmacol. 2014;155:801-9.

30. Bourjeily G, Danilack VA, Bublitz MH, Lipkind H, Muri J, Caldwell D, et al. Obstructive sleep apnea in pregnancy is associated with adverse maternal outcomes: a national cohort. Sleep Med. 2017;38:50-57.

31. Yuan T, Ma H, Liu W, Niesen DB, Shah N, Crews R, et al. Pomegranate's neuroprotective effects against Alzheimer's disease are mediated by urolithins, its ellagitannin-gut microbial derived metabolites. ACS Chem Neurosci. 2016;7:26-33.

32. Kujawska M, Jourdes M, Kurpik M, Szulc M, Szaefer H, Chmielarz P, et al. Neuroprotective effects of pomegranate juice against Parkinson's disease and presence of ellagitannins-derived metabolite-urolithin A-in the brain. Int J Mol Sci. 2019;21:202.

33. Verzelloni E, Pellacani C, Tagliazucchi D, Tagliaferri S, Calani L, Costa LG, et al. Antiglycative and neuroprotective activity of colon-derived polyphenol catabolites. Mol Nutr Food Res. 2011;55(Suppl 1):S35-43.

34. Gonzalez-Sarrias A, Nunez-Sanchez MA, Tomas-Barberan FA, Espin JC. Neuroprotective effects of bioavailable polyphenolderived metabolites against oxidative stress-induced cytotoxicity in human neuroblastoma SH-SY5Y cells. J Agric Food Chem. 2017;65:752-8.

35. Lee G, Park JS, Lee EJ, Ahn JH, Kim HS. Anti-inflammatory and antioxidant mechanisms of urolithin $\mathrm{B}$ in activated microglia. Phytomedicine. 2019;55:50-7.

36. Ryu D, Mouchiroud L, Andreux PA, Katsyuba E, Moullan N, Nicolet-Dit-Felix AA, et al. Urolithin A induces mitophagy and prolongs lifespan in C. elegans and increases muscle function in rodents. Nat Med. 2016;22:879-88.

37. Sun Y, Vashisht AA, Tchieu J, Wohlschlegel JA, Dreier L. Voltage-dependent anion channels (VDACs) recruit Parkin to defective mitochondria to promote mitochondrial autophagy. J Biol Chem. 2012;287:40652-60.

38. Cerda B, Periago P, Espin JC, Tomas-Barberan FA. Identification of urolithin a as a metabolite produced by human colon microflora from ellagic acid and related compounds. J Agric Food Chem. 2005;53:5571-6.

39. Zhao W, Shi F, Guo Z, Zhao J, Song X, Yang H. Metabolite of ellagitannins, urolithin A induces autophagy and inhibits 
metastasis in human sw620 colorectal cancer cells. Mol Carcinog. 2018;57:193-200.

40. Kasimsetty SG, Bialonska D, Reddy MK, Ma G, Khan SI, Ferreira D. Colon cancer chemopreventive activities of pomegranate ellagitannins and urolithins. J Agric Food Chem. 2010;58:2180-7.

41. Boakye YD, Groyer L, Heiss EH. An increased autophagic flux contributes to the anti-inflammatory potential of urolithin $\mathrm{A}$ in macrophages. Biochim Biophys Acta Gen Subj. 2018;1862:61-70.

42. Singh VP, Bali A, Singh N, Jaggi AS. Advanced glycation end products and diabetic complications. Korean J Physiol Pharm. 2014;18:1-14.

43. Dassanayaka S, Readnower RD, Salabei JK, Long BW, Aird AL, Zheng YT, et al. High glucose induces mitochondrial dysfunction independently of protein O-GlcNAcylation. Biochem J. 2015;467:115-26.

44. Banerjee PS, Ma J, Hart GW. Diabetes-associated dysregulation of O-GlcNAcylation in rat cardiac mitochondria. Proc Natl Acad Sci USA. 2015;112:6050-5.

45. Detaille D, Guigas B, Chauvin C, Batandier C, Fontaine E, Wiernsperger $\mathrm{N}$, et al. Metformin prevents high-glucose-induced endothelial cell death through a mitochondrial permeability transition-dependent process. Diabetes. 2005;54:2179-87.

46. Ji L, Liu F, Jing Z, Huang Q, Zhao Y, Cao H, et al. MICU1 alleviates diabetic cardiomyopathy through mitochondrial $\mathrm{Ca}^{2}$ ${ }^{+}$-dependent antioxidant response. Diabetes. 2017;66:1586-1600.

47. Zhang S, Al-Maghout T, Cao H, Pelzl L, Salker MS, Veldhoen M, et al. Gut bacterial metabolite urolithin A (UA) mitigates $\mathrm{Ca}^{2+}$ entry in $\mathrm{T}$ cells by regulating miR-10a-5p. Front Immunol. 2019;10:1737.

48. Bayle M, Neasta J, Dall'Asta M, Gautheron G, Virsolvy A, Quignard JF, et al. The ellagitannin metabolite urolithin C is a glucose-dependent regulator of insulin secretion through activation of L-type calcium channels. $\mathrm{Br} \mathrm{J}$ Pharm. 2019;176:4065-78.

49. Gong Z, Huang J, Xu B, Ou Z, Zhang L, Lin X, et al. Urolithin A attenuates memory impairment and neuroinflammation in APP/ PS1 mice. J Neuroinflammation. 2019;16:62.

50. Selma MV, Gonzalez-Sarrias A, Salas-Salvado J, Andres-Lacueva C, Alasalvar C, Orem A, et al. The gut microbiota metabolism of pomegranate or walnut ellagitannins yields two urolithinmetabotypes that correlate with cardiometabolic risk biomarkers: comparison between normoweight, overweight-obesity and metabolic syndrome. Clin Nutr. 2018;37:897-905.

51. Toney AM, Fan R, Xian Y, Chaidez V, Ramer-Tait AE, Chung S, et al. A gut metabolite, improves insulin sensitivity through augmentation of mitochondrial function and biogenesis. Obes (Silver Spring). 2019;27:612-20.

52. Greenberg CS, Birckbichler PJ, Rice RH. Transglutaminases: multifunctional cross-linking enzymes that stabilize tissues. FASEB J. 1991;5:3071-7.

53. Lorand L, Graham RM. Transglutaminases: crosslinking enzymes with pleiotropic functions. Nat Rev Mol Cell Biol. 2003;4:140-56.

54. Piacentini M, D'Eletto M, Falasca L, Farrace MG, Rodolfo C. Transglutaminase 2 at the crossroads between cell death and survival. Adv Enzymol Relat Areas Mol Biol. 2011;78:197-246.
55. Facchiano F, Facchiano A, Facchiano AM. The role of transglutaminase-2 and its substrates in human diseases. Front Biosci. 2006;11:1758-73.

56. Ludvigsen TP, Olsen LH, Pedersen HD, Christoffersen BO, Jensen LJ. Hyperglycemia-induced transcriptional regulation of ROCK1 and TGM2 expression is involved in small artery remodeling in obese diabetic Gottingen Minipigs. Clin Sci (Lond). 2019;133:2499-516.

57. Schelling JR. Tissue transglutaminase inhibition as treatment for diabetic glomerular scarring: it's good to be glueless. Kidney Int. 2009;76:363-5.

58. Dabir P, Marinic TE, Krukovets I, Stenina OI. Aryl hydrocarbon receptor is activated by glucose and regulates the thrombospondin-1 gene promoter in endothelial cells. Circ Res. 2008;102:1558-65.

59. Muku GE, Murray IA, Espin JC, Perdew GH. Urolithin A is a dietary microbiota-derived human aryl hydrocarbon receptor antagonist. Metabolites. 2018;8:86.

60. Andreola F, Calvisi DF, Elizondo G, Jakowlew SB, Mariano J, Gonzalez FJ, et al. Reversal of liver fibrosis in aryl hydrocarbon receptor null mice by dietary vitamin A depletion. Hepatology. 2004;39:157-66.

61. Singh R, Chandrashekharappa S, Bodduluri SR, Baby BV, Hegde B, Kotla NG, et al. Enhancement of the gut barrier integrity by a microbial metabolite through the Nrf2 pathway. Nat Commun. 2019;10:89.

62. Nukaya M, Lin BC, Glover E, Moran SM, Kennedy GD, Bradfield CA. The aryl hydrocarbon receptor-interacting protein (AIP) is required for dioxin-induced hepatotoxicity but not for the induction of the Cyp1a1 and Cyp1a2 genes. J Biol Chem. 2010;285:35599-605.

63. Stockinger B, Di Meglio P, Gialitakis M, Duarte JH. The aryl hydrocarbon receptor: multitasking in the immune system. Annu Rev Immunol. 2014;32:403-32.

64. Trivellin G, Korbonits M. AIP and its interacting partners. J Endocrinol. 2011;210:137-55.

65. Martino Adami PV, Nichtova Z, Weaver DB, Bartok A, Wisniewski T, Jones DR, et al. Perturbed mitochondria-ER contacts in live neurons that model the amyloid pathology of Alzheimer's disease. J Cell Sci. 2019;132:jcs229906.

66. Area-Gomez E, de Groof AJ, Boldogh I, Bird TD, Gibson GE, Koehler CM, et al. Presenilins are enriched in endoplasmic reticulum membranes associated with mitochondria. Am J Pathol. 2009;175:1810-6.

67. Calvo-Rodriguez M, Hernando-Perez E, Nunez L, Villalobos C. Amyloid $\beta$ oligomers increase ER-mitochondria $\mathrm{Ca}^{2+}$ cross talk in young hippocampal neurons and exacerbate aginginduced intracellular $\mathrm{Ca}^{2+}$ remodeling. Front Cell Neurosci. 2019;13:22.

68. Min B, Chung KC. New insight into transglutaminase 2 and link to neurodegenerative diseases. BMB Rep. 2018;51:5-13.

69. Norlund MA, Lee JM, Zainelli GM, Muma NA. Elevated transglutaminase-induced bonds in PHF tau in Alzheimer's disease. Brain Res. 1999;851:154-63.

70. Fa M, Orozco IJ, Francis YI, Saeed F, Gong Y, Arancio O. Preparation of oligomeric $\beta$-amyloid 1-42 and induction of synaptic plasticity impairment on hippocampal slices. J Vis Exp. 2010;2010:e1884. 


\section{Affiliations}

Hyun Jik Lee ${ }^{1,2} \cdot$ Young Hyun Jung ${ }^{3} \cdot$ Gee Euhn Choi ${ }^{3} \cdot$ Jun Sung Kim $\mathbb{D}^{3} \cdot$ Chang Woo Chae $^{3} \cdot$ Jae Ryong Lim ${ }^{3}$. Seo Yihl $\mathrm{Kim}^{3} \cdot$ Jee Hyeon Yoon ${ }^{3} \cdot \mathrm{Ji}$ Hyeon $\mathrm{Cho}^{3} \cdot$ Sei-Jung Lee $\mathbb{D i D}^{4} \cdot \mathrm{Ho}$ Jae $\mathrm{Han}^{3}$

1 Laboratory of Veterinary Physiology, College of Veterinary Medicine, Chungbuk National University, Cheongju, Chungbuk 28644, South Korea

2 Institute for Stem Cell and Regenerative Medicine (ISCRM), Chungbuk National University, Cheongju, Chungbuk 28644, South Korea

3 Department of Veterinary Physiology, College of Veterinary Medicine, Research Institute for Veterinary Science, and BK21
PLUS Program for Creative Veterinary Science Research, Seoul National University, Seoul 08826, South Korea

4 Department of Pharmaceutical Engineering, Daegu Haany University, Gyeongsan 38610, South Korea 